\title{
U-Pb Geochronology and Geochemistry of U-Rich Garnet from the Giant Beiya Gold-Polymetallic Deposit in SW China: Constraints on Skarn Mineralization Process
}

\author{
Yu Fu ${ }^{1,2}$, Xiaoming Sun ${ }^{1,2,3, *}$, Dengfeng $\mathrm{Li}^{1,2}$ and Hai Lin ${ }^{2,3}$ \\ 1 School of Marine Sciences, Sun Yat-sen University, Guangzhou 510006, China; \\ fuyu26@mail.sysu.edu.cn (Y.F.); lidf3@mail.sysu.edu.cn (D.L.) \\ 2 Guangdong Provincial Key Laboratory of Marine Resources and Coastal Engineering, Guangzhou 510275, \\ China; linhai7@mail2.sysu.edu.cn \\ 3 School of Earth Science and Engineering, Sun Yat-sen University, Guangzhou 510275, China \\ * Correspondence: eessxm@mail.sysu.edu.cn; Tel.: +86-20-8411-0968
}

Received: 30 January 2018; Accepted: 22 March 2018; Published: 23 March 2018

\begin{abstract}
The giant Beiya Au skarn deposit (over 300 tonnes Au metal reserve) is located in the middle part of the Jinshajiang-Ailaoshan alkaline porphyry metallogenic belt. The deposit is the largest Au skarn deposit and third largest $\mathrm{Au}$ deposit in China. In this paper, we present garnet $\mathrm{U}-\mathrm{Pb}$ ages and trace element geochemical data from two types of skarn-related U-rich garnet from Beiya, and discuss their implications on skarn metallogenesis. Based on optical characteristics and major element compositions, the older Grt I and the younger Grt II (both belong to the grossular-andradite solid solution) are distinguishable: Grt II ( $\left.\mathrm{Adr}_{69.0} \mathrm{Grs}_{26.8}-\mathrm{Adr}_{93.5} \mathrm{Grs}_{1.5}\right)$ is slightly richer in Fe than Grt I $\left(\mathrm{Adr}_{68.5} \mathrm{Grs}_{20.3}-\mathrm{Adr}_{86.3} \mathrm{Grs}_{3.4}\right)$, with the average $\mathrm{FeO}$ contents being $25.0 \%$ and $24.5 \%$, respectively. LA-ICP-MS garnet U-Pb dating yielded lower intercept ages of $35.8 \pm 0.8 \mathrm{Ma}(\mathrm{MSWD}=0.9, n=20$ ) and $34.0 \pm 2.1 \mathrm{Ma}$ (MSWD =1.2,n=16), respectively. These ages represent the timing of the garnet crystallization, and are consistent with published alteration ages within error, which suggests that the technique presented provides a reliable alternative in dating skarn alteration. Grt I has higher $\mathrm{U}$ content and lower Th/U ratio than Grt II, indicating a lower oxygen fugacity for the earlier skarn alteration. Grt II shows weak (or no) Eu anomaly and lower LREE/HREE ratios than those of Grt I, which indicates that Grt I and Grt II may have formed under a mildly acidic and nearly neutral condition, respectively. From the early prograde skarn (Grt I) to late prograde skarn (Grt II) stage, the ore-forming fluids may have changed from a relatively reduced acidic to a relatively oxidized neutral $\mathrm{pH}$ condition.
\end{abstract}

Keywords: giant Beiya gold skarn deposit; garnet; U-Pb dating; trace elements; Sanjiang region (SW China)

\section{Introduction}

Garnet is a common mineral in metamorphic rocks and hydrothermally-altered rocks such as skarn. Geochemical characteristics of garnet have been widely used to investigate complex magmatic, metamorphic and hydrothermal processes [1-7]. Dating of these processes could be achieved by garnet $\mathrm{Sm}-\mathrm{Nd}$ or Lu-Hf geochronology [8-10]. Grandite garnet has commonly higher U and Th concentrations than the other garnet types because of the substitution of $\mathrm{Fe}^{3+}$ for $\mathrm{Al}^{3+}$ (which facilitates $\mathrm{U}^{4+}$ incorporation [11]), making it suitable for U-Pb dating [8,12,13]. Laser ablation inductively coupled plasma mass spectrometry (LA-ICP-MS) U-Pb dating of grandite has the advantages of providing 
in-situ and rapid analyses, and has been widely applied to date various magmatic and hydrothermal processes $[14,15]$. Furthermore, garnet from different rock/ore-forming origins exhibits distinct major and trace element features, thus trace element geochemistry of garnet can serve as an indicator of its formation environment [5,16-19].

The giant Beiya Au-polymetallic skarn deposit is situated in the middle part of the Jinshajiang-Ailaoshan alkaline porphyry metallogenic belt. The deposit is the largest Au skarn deposit in China with significant amounts of $\mathrm{Fe}, \mathrm{Cu}, \mathrm{Pb}, \mathrm{Zn}$ and $\mathrm{Ag}$. The Jinshajiang-Ailaoshan alkaline porphyry metallogenic belt is an important $\mathrm{Cu}-\mathrm{Au}$ province in SW China, and contains a large number of deposits that are space-time associated with the Cenozoic (Himalayan) alkaline magmatism [20-23]. Ore deposit geology, ore fluid composition and metallogenic studies of the Beiya Au mineralization all suggest that the Beiya deposit resembles typical porphyry-related Au skarn deposits worldwide [24-29].

Garnet is a common mineral in the Beiya skarn ores, and provides a suitable target for geochronology and geochemistry study. In this study, we present new $\mathrm{U}-\mathrm{Pb}$ and trace element data for two U-rich garnet types from Beiya. Our new data are integrated with published results to discuss the ore-forming environment and constrain the Beiya skarn mineralization processes. Moreover, we examine the precision and effectiveness of the LA-ICP-MS garnet U-Pb dating technique, and its applications on constraining the timing of skarn mineralization.

\section{Geological Setting and Ore Geology}

\subsection{Regional Geology}

The Beiya deposit is the largest gold deposit in the region and ranks as the third largest gold deposit in China, with estimated reserves of 130.8 million tonnes (Mt) Au ore at $2.47 \mathrm{~g} / \mathrm{t}, 170 \mathrm{Mt} \mathrm{Fe}$ ore at $33.3 \mathrm{wt} \%$, and $125 \mathrm{Mt} \mathrm{Cu}$ ore at $0.52 \mathrm{wt} \%$, along with considerable amounts of $\mathrm{Pb}, \mathrm{Zn}$ and Ag [30,31]. The Beiya skarn gold-polymetallic deposit is located to the east of the Jinshajiang suture (western margin of the Yangtze Craton) in SW China (Figure 1), and situated in the middle part of the Jinshajiang-Ailaoshan alkaline porphyry metallogenic belt [32]. Among the many porphyry-related deposits in the belt, Beiya is the most representative with regards to scale, and covers an area of $\sim 800 \mathrm{~km}^{2}$. The deposit is located at the limbs of the NS-trending Beiya syncline, and comprises six ore segments, namely: Weiganpo, Bijiashan, Guogaishan, Wandongshan, Hongnitang and Jingouba. These segments are divided into two zones, with the former three and latter three located on the eastern and western limb of the Beiya syncline, respectively (Figure 2a). Among these segments, the Wandongshan section (contains mainly the KT52 orebody) is the largest and contains most of the Au resources (99 Mt @2.61 g/t Au [24]). 


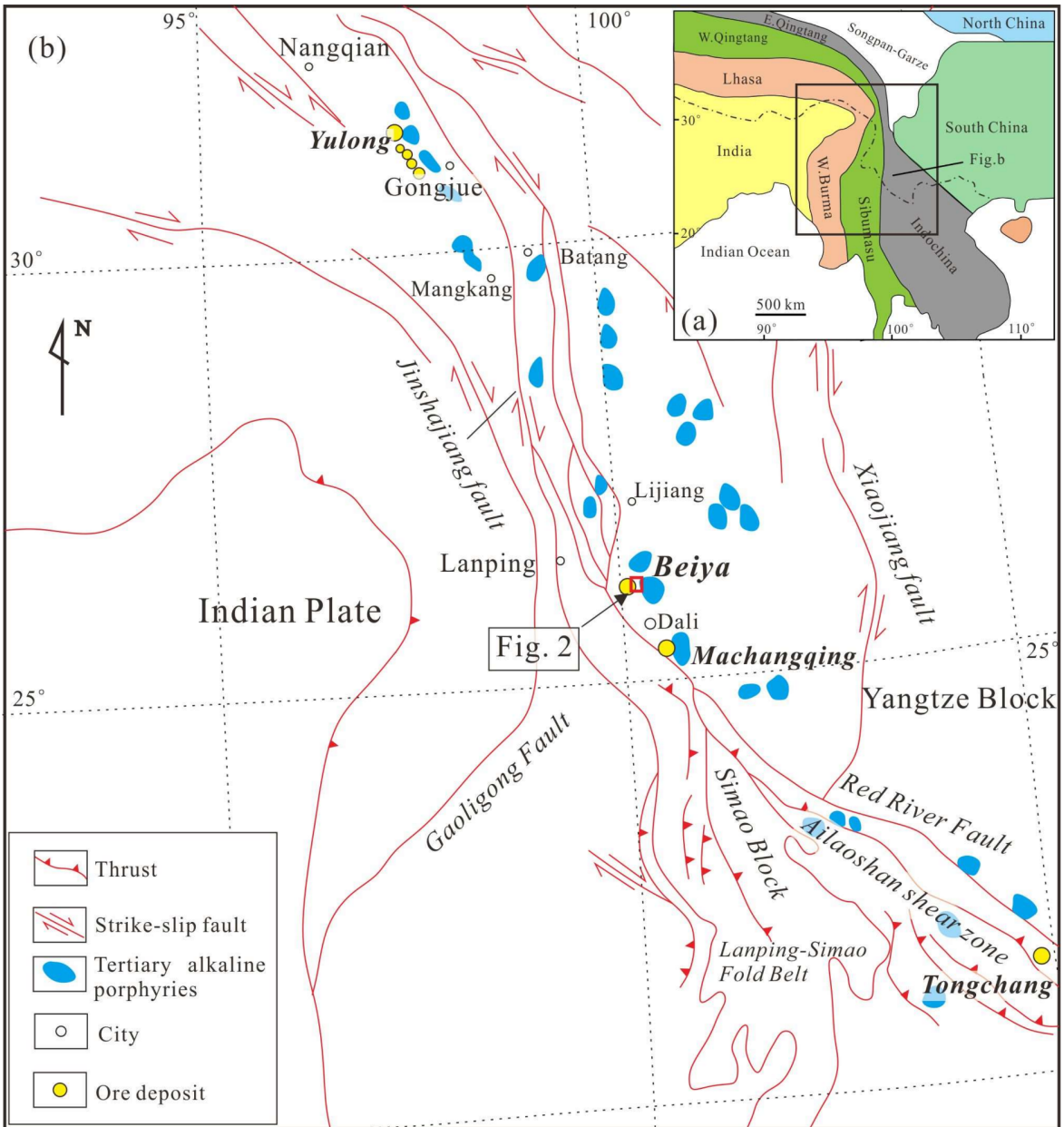

Figure 1. (a) Distribution of principal continental blocks of the East Tethyan belt. (b) Tectonic setting of the Beiya deposit. Modified after [33-35].
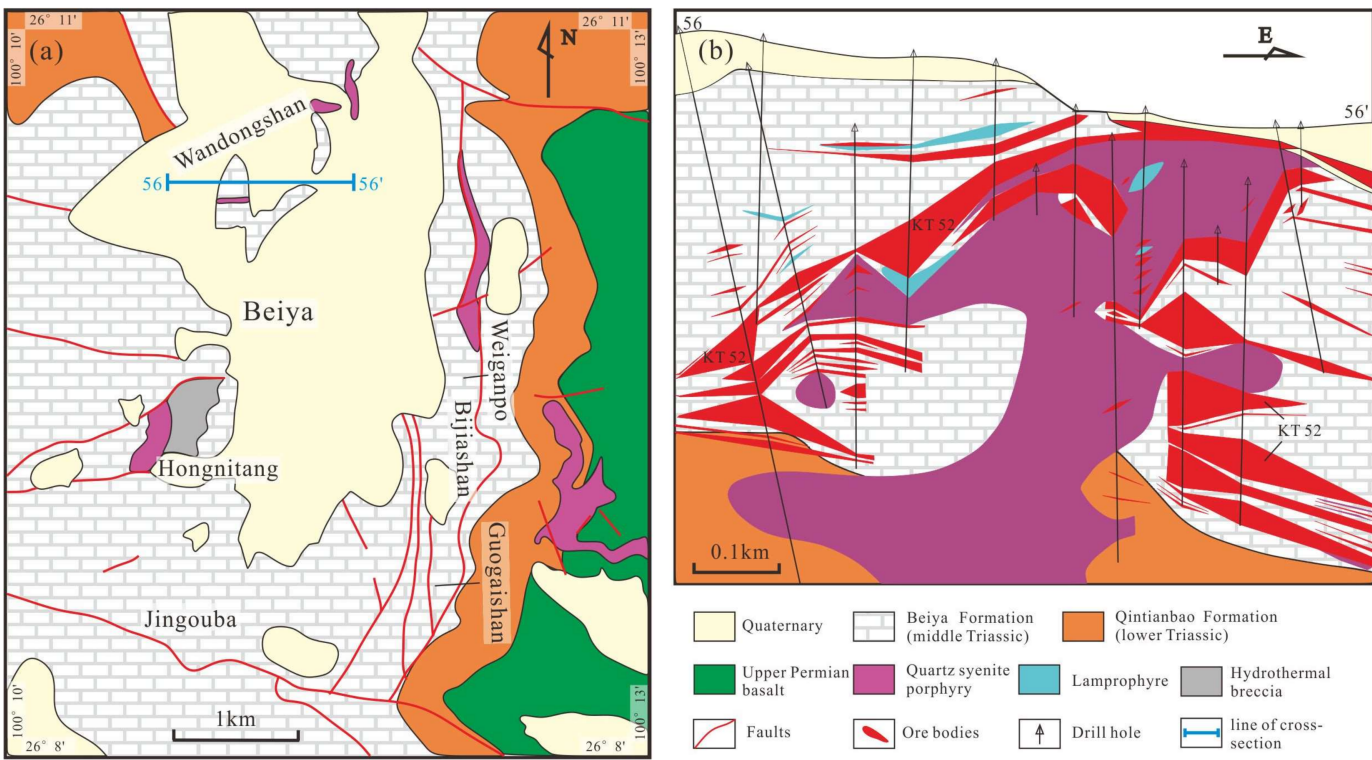

Figure 2. (a) Geologic map and (b) cross-section of the Beiya deposit. Modified after [36,37]. 
Exposed stratigraphy at Beiya comprises the Lower Triassic Qingtianbao Formation (175-350 m thick), the Middle Triassic Beiya Formation and Quaternary sedimentary rocks. The Lower Triassic Qingtianbao Formation is exposed mainly in the eastern part of the ore district (Figure 2a), which contains arkose, hornfelsed greywacke and sandstone with basaltic volcaniclastic rocks; in contrast, the Middle Triassic Beiya Formation occupies most of the ore distinct and contains dolomitic, ferruginous, bioclastic and argillaceous limestone (138-531 m thick), which serves as the main ore host. Outcropping igneous rocks in and around the ore district include the Upper Permian Emeishan flood basalt and the Cenozoic alkaline porphyries. The Emeishan flood basalt is found in the southeastern part of the mining area. The Cenozoic alkaline porphyries include (quartz) syenite-, biotite-K-feldsparand quartz-albite porphyries [25,27,38-40]. Although these porphyries are widespread in the region, they are relatively rare within the ore district but are considered closely related to the mineralization.

There are two main fault systems in the district, i.e., $\mathrm{N}$ - and E-trending. The former is dominant, and includes the Maanshan Fault (a branch of the Jinshajiang-Honghe strike-slip fault system) that extends across western Beiya, and its secondary structures are interpreted to control the porphyry/orebody emplacement in the Wandongshan and Hongnitang ore segments [41]. The E-striking fault system is likely post-mineralized and has probably truncated/destroyed some orebodies [42].

\subsection{Ore Deposit Geology}

Porphyries at Beiya include mainly quartz syenite, quartz monzonite, biotite-orthoclase, quartz-albite and a group of lamprophyre dikes. Most of them were emplaced at ca. 36.9 to 33.3 Ma [23-25,27,43-47], except for the quartz-albite porphyry (65 Ma) and biotite-orthoclase porphyry (3.8 Ma) [40]. The Beiya quartz syenite porphyry is thought to be ore-related, and was dated as $36.1 \pm 0.43 \mathrm{Ma}$ [23] by zircon $\mathrm{U}-\mathrm{Pb}$, providing the minimum age of the skarn alteration. The Beiya skarn orebodies occur mostly in and/or along the intrusive contacts with the carbonate rocks of the Beiya Formation. Some stratiform orebodies are locally distributed along flat interlayer fractures and breccia zones within the Beiya Formation carbonates, as well as along the contact between the carbonates and the underlying Qingtianbao Formation sandstone. Vein-like orebodies occur within the porphyritic intrusions, and laterite-hosted orebodies have also been documented [41]. Previous studies have documented three mineralization styles at Beiya (i.e., porphyry, skarn and supergene), among which skarn mineralization is the most important.

Skarn mineralization at Beiya comprises three stages: (1) the prograde skarn is closely associated with the porphyry emplacements, and comprises anhydrous minerals such as garnet and pyroxene; (2) the retrograde alteration stage was hydrothermal titanite U-Pb dated to be $33.1 \pm 1.0 \mathrm{Ma}$ [48], and contains predominantly hydrous minerals (including epidote, biotite and chlorite), magnetite, titanite, feldspar with minor scheelite and fluorite. Magnetite is the main ore mineral of this stage and formed massive orebodies; (3) the quartz-sulfide stage was molybdenite Re-Os dated to be $36.8 \pm 0.5 \mathrm{Ma}$ and $34.7 \pm 1.6 \mathrm{Ma}[23,27]$, and contains mainly pyrite, chalcopyrite, pyrrhotite and molybdenite. Although the mineral assemblage indicated that the quartz-sulfide stage is later than the retrograde stage, the former age $(36.8 \pm 0.5 \mathrm{Ma})$ is older than the age of the retrograde stage $(33.1 \pm 1.0 \mathrm{Ma})$ within the associated uncertainties. Possibly it is because that the samples investigated previously are from different alteration zones. Gold at Beiya occurs mainly as native gold and electrum in fractures and/or as vein-infill with pyrite, magnetite, limonite and quartz, and is precipitated in the late retrograde alteration and quartz-sulfide stage.

\section{Samples and Analytical Methods}

Most garnet grains in the Beiya deposit are low U garnet. In this study, U-rich garnet samples were selected after preliminary trace element analyses (over 200 points in different garnet samples). Two U-rich garnet-bearing rock samples for the LA-ICP-MS U-Pb dating and elemental analysis were collected from the 84ZK14 drill cores in the mineralized skarn at the Wandongshan ore segment. 
Sample W281 contains mainly garnet with minor chlorite, magnetite, pyrite and chalcopyrite (Figure 3a), whereas sample W297 occurs in an assemblage of garnet, epidote, chlorite and magnetite, with minor pyrite and chalcopyrite (Figure 3b). Thin sections were prepared for all the samples, and were studied with optical microscopy and back-scattered electron (BSE) imaging, using a Zeiss SIGMA field-emission Scanning Electron Microscope (SEM) at the School of Earth Science and Engineering, Sun Yat-sen University (Guangzhou, China). Prior to the LA-ICP-MS analysis, major element abundance of the garnet samples was determined on thin sections using a 1720 electron probe micro-analyzer (EPMA) (Shimadzu Corporation, Kyoto, Japan) at the School of Geosciences and Info-Physics of the Central South University (Changsha, China). Operating conditions include $15 \mathrm{kV}$ accelerating voltage, $10 \mathrm{nA}$ beam current and a beam diameter of $2 \mu \mathrm{m}$. In-situ U-Pb dating and trace element analyses of garnet were performed by LA-ICP-MS at the Key Laboratory of Marine Resources and Coastal Engineering, Sun Yat-sen University. The analyses were performed using an ArF excimer laser ablation system (GeoLasPro) coupled with an Agilent 7700x ICP-MS. A $32 \mu \mathrm{m}$ (for trace element analysis) and $44 \mu \mathrm{m}$ (for U-Pb dating) spot sizes were used with an energy density of $5 \mathrm{~J} / \mathrm{cm}^{2}$ and a repetition rate of $5 \mathrm{~Hz}$. The trace element compositions of garnet were calibrated against the glass standard NIST610 [49], using Si determined by EPMA as the internal standard. Zircon 91500 [50] was used as the external standard for the U-Pb dating. Time-dependent drifts of U-Th-Pb isotopic ratios were corrected using a linear interpolation (with time) for every ten analyses, based on the variations of 91500. Zircon Plěsovice [51] and garnet QC04 [14] were used as the secondary standards for data quality control and the obtained values are consistent with the recommended values within the associated uncertainties. Each analysis consists of a $20 \mathrm{~s}$ background measurement (laser-off) followed by $45 \mathrm{~s}$ of data acquisition. Data reduction was performed using ICPMSDataCal software [52]. ISOPLOT 3.0 software [53] was used to construct the Tera-Wasserburg diagram.

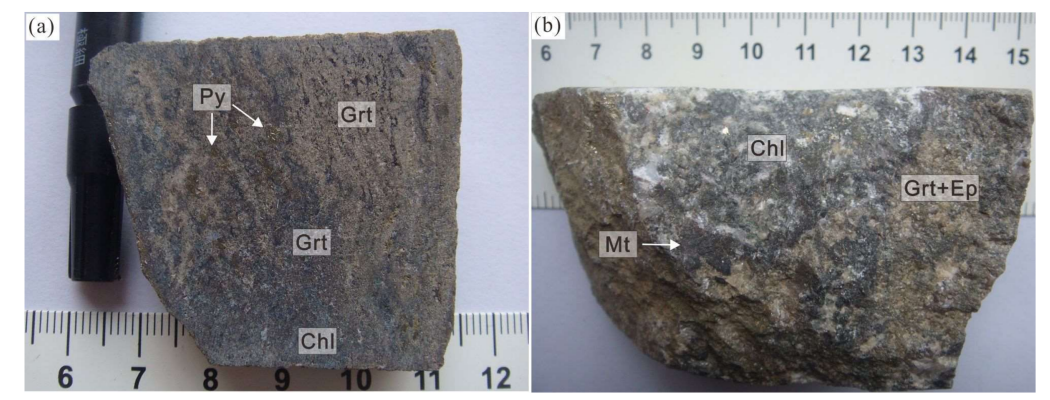

Figure 3. Hand-specimen photographs of the two garnet-bearing samples from Beiya, (a) W281, (b) W297. Abbreviations: Chl, chlorite; Ep, epidote; Grt, garnet; Mt, magnetite; Py, pyrite.

\section{Results}

\subsection{Garnet Petrography}

Based on the mineral assemblage and texture, the U-rich garnet from W297 and W281 may represent for two distinct types of garnet that are well developed in the Beiya skarn deposit. Garnet type I (abbreviated as Grt I; e.g., sample W297) is characterized by coarse-grained (0.3-5 mm diameter), anisotropic and anhedral to subhedral texture (Figure 4a-e). Grt I grains exhibit polysynthetic twinning under the cross-polarized light (Figure $4 \mathrm{~b}-\mathrm{d}$ ), most of which are cracked and filled with or enclosed by biotite, chlorite, plagioclase and pyroxene (Figure $4 \mathrm{a}-\mathrm{d}$ ). Some cracks in Grt I grains are filled by magnetite (Figure 4e). Garnet type II (abbreviated as Grt II; e.g., sample W281) grains are euhedral, isotropic and medium to fine-grained (0.05-2 mm diameter) (Figure $4 \mathrm{f}-\mathrm{g}$ ). Most of Grt II grains are well-preserved with less fractures compared with Grt I. Some interstices of Grt II grains are filled by the hydrous minerals such as epidote (Figure $4 \mathrm{f}-\mathrm{g}$ ). There are also minor garnet pieces (Grt I) preserved showing polysynthetic twinning extinction character in W281 (Figure 4h), and 
they were commonly enclosed by the garnet with complete extinction texture (Grt II). Similarly, some garnet grains exhibit core-rim textures (Figure 4i), with the cores cracked and filled with chlorite and magnetite and the rims well-preserved with less fractures, which probably represent for Grt I and Grt II, respectively. The paragenetic relationships between the two types of garnet indicate that there might be two generations of garnet and Grt I probably formed earlier than Grt II.

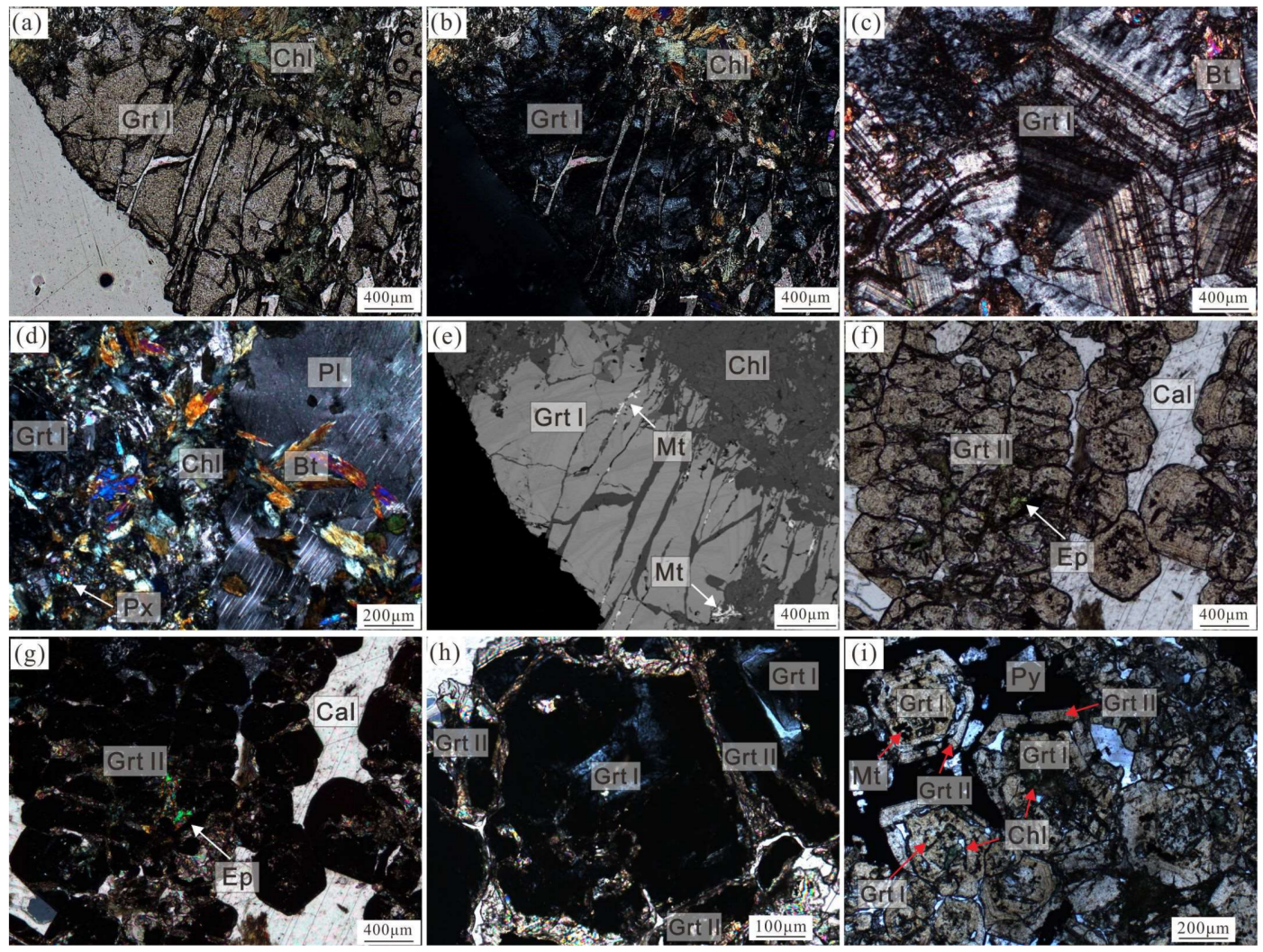

Figure 4. Photomicrographs and BSE image of the garnet samples from the Beiya deposit. (a-e) photomicrographs and BSE image showing the occurrence of Grt I (W297) ((a) plane-polarized light; (b-d) cross-polarized light; (e) BSE image); (f,g) photomicrographs of Grt II (W281) ((f) plane-polarized light; (g) cross-polarized light); (h,i) photomicrographs showing the paragenetic relationships of Grt I and Grt II ((h) cross-polarized light; (i) plane-polarized light). Abbreviations: Bt, biotite; Cal, calcite; Chl, chlorite; Ep, epidote; Grt, garnet; Mt, magnetite; Pl, plagioclase; Py, pyrite; Px, pyroxene.

\subsection{Major and Trace Elements of Garnet}

Major element compositions of the two Beiya garnet types are summarized in Table 1. Grt I (W297) has uniform $\mathrm{SiO}_{2}$ (37.5-39.1 wt \%) and $\mathrm{CaO}$ (31.6-32.7 wt \%), with $\mathrm{Al}_{2} \mathrm{O}_{3}$ and $\mathrm{FeO}$ concentrations of 0.6-3.8 wt \% and 22.8-26.4 wt \%, respectively. Grt II (W281) also exhibits uniform $\mathrm{SiO}_{2}(36.5-38.3 \mathrm{wt} \%)$ and $\mathrm{CaO}$ (32.3-34.5 wt \%), with $\mathrm{Al}_{2} \mathrm{O}_{3}$ and $\mathrm{FeO}$ concentrations of 0.3-5.7 wt \% and 20.6-28.9 wt $\%$, respectively. Grt II exhibits slightly higher FeO content than Grt I with the average values of $25.0 \%$ and $24.5 \%$, respectively. As shown in Figure 5, the Beiya garnet samples belong mostly to the grossular-andradite solid solution. Grt I ranges in composition from $\operatorname{Adr}_{68.5} \mathrm{Grs}_{20.3}$ to $\mathrm{Adr}_{86.3} \mathrm{Grs}_{3.4}$, with moderate amounts of pyralspite (almandine + spessartine + pyrope, average: 9.5\%). Grt II has lower pyralspite content (average: 3.7\%) than Grt I (Figure 5), and ranges in composition from $\operatorname{Adr}_{69.0} \mathrm{Grs}_{26.8}$ to $\mathrm{Adr}_{93.5} \mathrm{Grs}_{1.5}$. 
Table 1. Representative electron probe micro-analyzer (EPMA) results of garnets from the Beiya deposit.

\begin{tabular}{|c|c|c|c|c|c|c|c|c|c|c|c|c|c|c|c|c|c|c|c|}
\hline \multirow{2}{*}{$\begin{array}{c}\text { Sample } \\
\text { Spot No. }\end{array}$} & \multicolumn{19}{|c|}{ W297 (Grt I) } \\
\hline & 1 & 2 & 3 & 4 & 5 & 6 & 7 & 8 & 9 & 10 & 11 & 12 & 13 & 14 & 15 & 16 & 17 & 18 & 19 \\
\hline $\mathrm{SiO}_{2}(\mathrm{wt} \%)$ & 38.2 & 37.8 & 38.0 & 38.5 & 38.0 & 37.5 & 37.5 & 37.6 & 38.3 & 37.8 & 37.8 & 38.6 & 38.3 & 37.7 & 38.1 & 39.0 & 38.8 & 39.1 & 39.0 \\
\hline $\mathrm{Al}_{2} \mathrm{O}_{3}$ & 2.76 & 2.73 & 3.40 & 3.32 & 2.06 & 1.86 & 3.49 & 1.16 & 2.30 & 0.61 & 0.90 & 3.40 & 2.20 & 2.65 & 0.83 & 3.75 & 2.37 & 1.43 & 3.76 \\
\hline $\mathrm{FeO}$ & 23.9 & 23.8 & 23.0 & 23.4 & 25.0 & 25.0 & 23.0 & 26.1 & 24.8 & 26.3 & 26.4 & 23.4 & 24.1 & 24.1 & 26.4 & 22.9 & 25.0 & 26.0 & 22.8 \\
\hline $\mathrm{MnO}$ & 0.18 & 0.23 & 0.24 & 0.17 & 0.13 & 0.18 & 0.41 & 0.33 & 0.36 & 0.37 & 0.33 & 0.38 & 0.32 & 0.21 & 0.23 & 0.20 & 0.21 & 0.18 & 0.22 \\
\hline $\mathrm{MgO}$ & 0.05 & 0.08 & 0.06 & 0.03 & 0.06 & 0.06 & 0.10 & 0.09 & 0.14 & 0.10 & 0.07 & 0.11 & 0.12 & 0.06 & 0.07 & 0.02 & 0.03 & 0.03 & 0.02 \\
\hline $\mathrm{CaO}$ & 32.4 & 32.3 & 32.7 & 32.6 & 32.2 & 32.6 & 32.3 & 32.0 & 32.2 & 31.7 & 31.6 & 32.3 & 31.9 & 32.4 & 32.1 & 32.6 & 32.1 & 32.4 & 32.3 \\
\hline Total & 97.5 & 97.0 & 97.3 & 98.0 & 97.4 & 97.2 & 96.8 & 97.3 & 98.1 & 96.8 & 97.1 & 98.1 & 96.9 & 97.1 & 97.8 & 98.5 & 98.5 & 99.1 & 98.1 \\
\hline
\end{tabular}

Cations on the basis of 12 oxygens

$\begin{array}{llllllllllllllllllll}\mathrm{Si} & 3.172 & 3.157 & 3.153 & 3.172 & 3.169 & 3.134 & 3.126 & 3.147 & 3.169 & 3.190 & 3.175 & 3.175 & 3.205 & 3.144 & 3.177 & 3.196 & 3.194 & 3.209 & 3.204\end{array}$

Ti $\quad \begin{array}{llllllllllllllllllllll} & 0.000 & 0.000 & 0.000 & 0.000 & 0.000 & 0.000 & 0.000 & 0.000 & 0.000 & 0.000 & 0.000 & 0.000 & 0.000 & 0.000 & 0.000 & 0.000 & 0.000 & 0.000 & 0.000\end{array}$

$\begin{array}{llllllllllllllllllll}\text { Al } & 0.270 & 0.269 & 0.332 & 0.323 & 0.202 & 0.183 & 0.343 & 0.115 & 0.224 & 0.061 & 0.089 & 0.330 & 0.217 & 0.260 & 0.082 & 0.362 & 0.230 & 0.139 & 0.364\end{array}$

$\begin{array}{llllllllllllllllllll}\mathrm{Cr} & 0.000 & 0.000 & 0.000 & 0.000 & 0.000 & 0.000 & 0.000 & 0.000 & 0.000 & 0.000 & 0.000 & 0.000 & 0.000 & 0.000 & 0.000 & 0.000 & 0.000 & 0.000 & 0.000\end{array}$

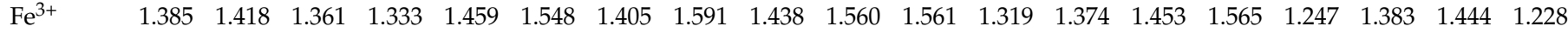

$\begin{array}{llllllllllllllllllll}\mathrm{Fe}^{2+} & 0.275 & 0.243 & 0.230 & 0.280 & 0.279 & 0.200 & 0.200 & 0.239 & 0.276 & 0.291 & 0.294 & 0.289 & 0.311 & 0.230 & 0.281 & 0.319 & 0.338 & 0.341 & 0.342\end{array}$

$\begin{array}{llllllllllllllllllll}\text { Mn } & 0.013 & 0.016 & 0.017 & 0.012 & 0.009 & 0.013 & 0.029 & 0.024 & 0.025 & 0.026 & 0.024 & 0.027 & 0.022 & 0.015 & 0.016 & 0.014 & 0.015 & 0.012 & 0.016\end{array}$

$\begin{array}{llllllllllllllllllllll}\mathrm{Mg} & 0.006 & 0.010 & 0.007 & 0.004 & 0.007 & 0.007 & 0.013 & 0.011 & 0.017 & 0.013 & 0.008 & 0.013 & 0.015 & 0.008 & 0.009 & 0.003 & 0.003 & 0.003 & 0.003\end{array}$

$\begin{array}{lllllllllllllllllllll}\mathrm{Ca} & 2.879 & 2.887 & 2.900 & 2.877 & 2.874 & 2.915 & 2.884 & 2.873 & 2.850 & 2.859 & 2.849 & 2.847 & 2.856 & 2.891 & 2.872 & 2.860 & 2.838 & 2.852 & 2.844\end{array}$

$\begin{array}{llllllllllllllllllll}\text { Total } & 8.000 & 8.000 & 8.000 & 8.000 & 8.000 & 8.000 & 8.000 & 8.000 & 8.000 & 8.000 & 8.000 & 8.000 & 8.000 & 8.000 & 8.000 & 8.000 & 8.000 & 8.000 & 8.000\end{array}$

$\begin{array}{llllllllllllllllllll}\text { Almandine } & 8.7 & 7.7 & 7.3 & 8.8 & 8.8 & 6.4 & 6.4 & 7.6 & 8.7 & 9.1 & 9.3 & 9.1 & 9.7 & 7.3 & 8.8 & 10.0 & 10.6 & 10.6 & 10.7\end{array}$

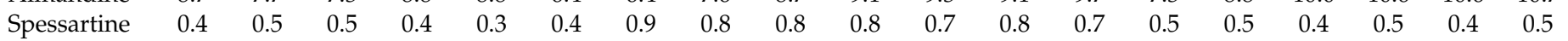

$\begin{array}{llllllllllllllllllll}\text { Pyrope } & 0.2 & 0.3 & 0.2 & 0.1 & 0.2 & 0.2 & 0.4 & 0.4 & 0.5 & 0.4 & 0.3 & 0.4 & 0.5 & 0.2 & 0.3 & 0.1 & 0.1 & 0.1 & 0.1\end{array}$

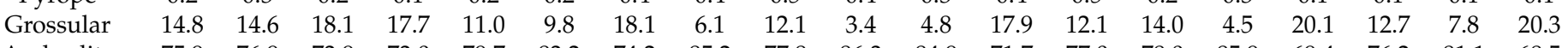

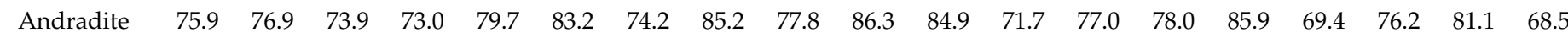

\begin{tabular}{|c|c|c|c|c|c|c|c|c|c|c|c|c|c|c|c|c|c|c|c|c|c|c|c|c|}
\hline Sample & W28 & $\overrightarrow{S I}$ & & & & & & & & & & & & & & & & & & & & & & \\
\hline Spot No. & 1 & 2 & 3 & 4 & 5 & 6 & 7 & 8 & 9 & 10 & 11 & 12 & 13 & 14 & 15 & 16 & 17 & 18 & 19 & 20 & 21 & 22 & 23 & 24 \\
\hline $\mathrm{SiO}_{2}(\mathrm{wt} \%)$ & 37.3 & 37.7 & 36.7 & 36.5 & 37.1 & 36.5 & 36.7 & 37.0 & 37.6 & 36.9 & 37.8 & 37.9 & 38.3 & 36.8 & 37.3 & 37.0 & 37.2 & 38.1 & 38.0 & 37.7 & 37.8 & 37.2 & 37.4 & 37.0 \\
\hline & 5 & 3.58 & 1.34 & 2.28 & 1.73 & 1.40 & 1.14 & 3.00 & 5.14 & 2.51 & 3.93 & 4.03 & 5.01 & 1.5 & 2.16 & 2.14 & 2.65 & 4.38 & 5.66 & 4.20 & 5.10 & .74 & .82 & 0.30 \\
\hline & & 0.37 & 0.31 & 0.18 & 0.1 & 0.20 & 0.15 & 0.17 & 0.25 & 0.37 & 0.28 & 0.32 & 0.29 & 0.33 & 0.28 & 0.32 & 0.27 & 0.37 & 0.46 & 0.34 & 0.47 & 0.29 & 0.28 & 0.23 \\
\hline $\mathrm{MgO}$ & & 0.17 & 0.05 & 0.04 & 0.13 & 0.12 & 0.02 & 0.09 & 0.13 & 0.24 & 0.05 & 0.22 & 0.10 & 0.29 & 0.17 & 0.26 & 0.17 & 0.08 & 0.13 & 0.05 & 0.17 & 0.04 & 0.07 & 0.07 \\
\hline $\mathrm{CaO}$ & & 33.3 & 32.7 & 33.1 & 33.3 & 33.4 & 33.6 & 33.6 & 34.3 & 33.4 & 34.5 & 34.2 & 34.3 & 33.0 & 33. & 32 & & 33.3 & & 34.3 & 33.9 & 33.2 & 33.6 & 32.8 \\
\hline Total & 98.0 & 99.7 & 98.0 & 97.8 & 99.1 & 97.7 & 98.5 & 98.9 & 99.3 & 98.8 & 100.0 & 100.2 & 100.3 & 99.0 & 99.5 & 98.3 & 100.1 & 100.1 & 100.1 & 100.3 & 99.7 & 99.6 & 100.0 & 99.2 \\
\hline
\end{tabular}

Cations on the basis of 12 oxygens

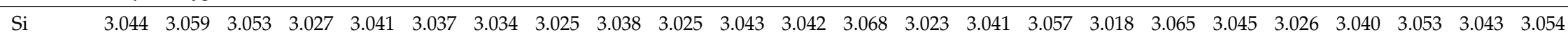


Table 1. Cont.

\begin{tabular}{|c|c|c|c|c|c|c|c|c|c|c|c|c|c|c|c|c|c|c|c|c|c|c|c|c|}
\hline \multirow{2}{*}{$\begin{array}{c}\text { Sample } \\
\text { Spot No. }\end{array}$} & \multicolumn{24}{|c|}{ W281 (Grt II) } \\
\hline & 1 & 2 & 3 & 4 & 5 & 6 & 7 & 8 & 9 & 10 & 11 & 12 & 13 & 14 & 15 & 16 & 17 & 18 & 19 & 20 & 21 & 22 & 23 & 24 \\
\hline \multicolumn{25}{|c|}{ Cations on the basis of 12 oxygens } \\
\hline $\mathrm{Ti}$ & 0.000 & 0.000 & 0.000 & 0.000 & 0.000 & 0.000 & 0.000 & 0.000 & 0.000 & 0.000 & 0.000 & 0.000 & 0.000 & 0.000 & 0.000 & 0.000 & 0.000 & 0.000 & 0.000 & 0.000 & 0.000 & 0.000 & 0.000 & 0.000 \\
\hline $\mathrm{Al}$ & 0.546 & 0.342 & 0.131 & 0.223 & 0.167 & 0.137 & 0.111 & 0.290 & 0.489 & 0.243 & 0.373 & 0.382 & 0.473 & 0.147 & 0.207 & 0.209 & 0.253 & 0.416 & 0.534 & 0.397 & 0.484 & 0.072 & 0.174 & 0.029 \\
\hline $\mathrm{Cr}$ & 0.000 & 0.000 & 0.000 & 0.000 & 0.000 & 0.000 & 0.000 & 0.000 & 0.000 & 0.000 & 0.000 & 0.000 & 0.000 & 0.000 & 0.000 & 0.000 & 0.000 & 0.000 & 0.000 & 0.000 & 0.000 & 0.000 & 0.000 & 0.000 \\
\hline $\mathrm{Fe}^{3+}$ & 1.365 & 1.541 & 1.763 & 1.722 & 1.750 & 1.788 & 1.822 & 1.660 & 1.436 & 1.707 & 1.542 & 1.535 & 1.392 & 1.806 & 1.710 & 1.678 & 1.710 & 1.454 & 1.376 & 1.551 & 1.436 & 1.821 & 1.740 & 1.863 \\
\hline $\mathrm{Fe}^{2+}$ & 0.042 & 0.121 & 0.109 & 0.069 & 0.084 & 0.031 & 0.039 & 0.055 & 0.038 & 0.036 & 0.043 & 0.050 & 0.094 & 0.058 & 0.083 & 0.140 & 0.071 & 0.156 & 0.081 & 0.041 & 0.061 & 0.113 & 0.090 & 0.130 \\
\hline $\mathrm{Mn}$ & 0.026 & 0.025 & 0.022 & 0.013 & 0.012 & 0.014 & 0.010 & 0.012 & 0.017 & 0.026 & 0.019 & 0.022 & 0.019 & 0.023 & 0.019 & 0.022 & 0.019 & 0.025 & 0.031 & 0.023 & 0.032 & 0.020 & 0.019 & 0.016 \\
\hline $\mathrm{Mg}$ & 0.023 & 0.021 & 0.007 & 0.005 & 0.016 & 0.015 & 0.003 & 0.010 & 0.016 & 0.029 & 0.006 & 0.026 & 0.012 & 0.036 & 0.021 & 0.032 & 0.021 & 0.010 & 0.016 & 0.006 & 0.020 & 0.004 & 0.008 & 0.008 \\
\hline $\mathrm{Ca}$ & 2.953 & 2.892 & 2.916 & 2.941 & 2.929 & 2.977 & 2.982 & 2.948 & 2.966 & 2.933 & 2.974 & 2.944 & 2.942 & 2.906 & 2.919 & 2.862 & 2.908 & 2.874 & 2.917 & 2.956 & 2.927 & 2.916 & 2.926 & 2.900 \\
\hline Total & 8.000 & 8.000 & 8.000 & 8.000 & 8.000 & 8.000 & 8.000 & 8.000 & 8.000 & 8.000 & 8.000 & 8.000 & 8.000 & 8.000 & 8.000 & 8.000 & 8.000 & 8.000 & 8.000 & 8.000 & 8.000 & 8.000 & 8.000 & 8.000 \\
\hline Almandine & 1.4 & 3.9 & 3.6 & 2.3 & 2.8 & 1.0 & 1.3 & 1.8 & 1.3 & 1.2 & 1.4 & 1.6 & 3.1 & 1.9 & 2.7 & 4.6 & 2.3 & 5.1 & 2.7 & 1.4 & 2.0 & 3.7 & 2.9 & 4.2 \\
\hline Spessartine & 0.8 & 0.8 & 0.7 & 0.4 & 0.4 & 0.5 & 0.3 & 0.4 & 0.6 & 0.9 & 0.6 & 0.7 & 0.6 & 0.8 & 0.6 & 0.7 & 0.6 & 0.8 & 1.0 & 0.8 & 1.0 & 0.7 & 0.6 & 0.5 \\
\hline Pyrope & 0.8 & 0.7 & 0.2 & 0.2 & 0.5 & 0.5 & 0.1 & 0.3 & 0.5 & 1.0 & 0.2 & 0.9 & 0.4 & 1.2 & 0.7 & 1.0 & 0.7 & 0.3 & 0.5 & 0.2 & 0.7 & 0.1 & 0.3 & 0.3 \\
\hline Grossular & 27.7 & 17.2 & 6.6 & 11.1 & 8.4 & 7.0 & 5.6 & 14.5 & 24.8 & 12.1 & 19.0 & 19.3 & 24.3 & 7.3 & 10.4 & 10.4 & 12.4 & 20.9 & 26.8 & 19.9 & 24.3 & 3.6 & 8.7 & 1.5 \\
\hline Andradite & 69.3 & 77.4 & 88.9 & 86.0 & 87.9 & 91.0 & 92.7 & 83.0 & 72.8 & 84.9 & 78.7 & 77.5 & 71.6 & 88.9 & 85.6 & 83.3 & 83.9 & 72.9 & 69.0 & 77.7 & 72.0 & 91.9 & 87.4 & 93.5 \\
\hline
\end{tabular}




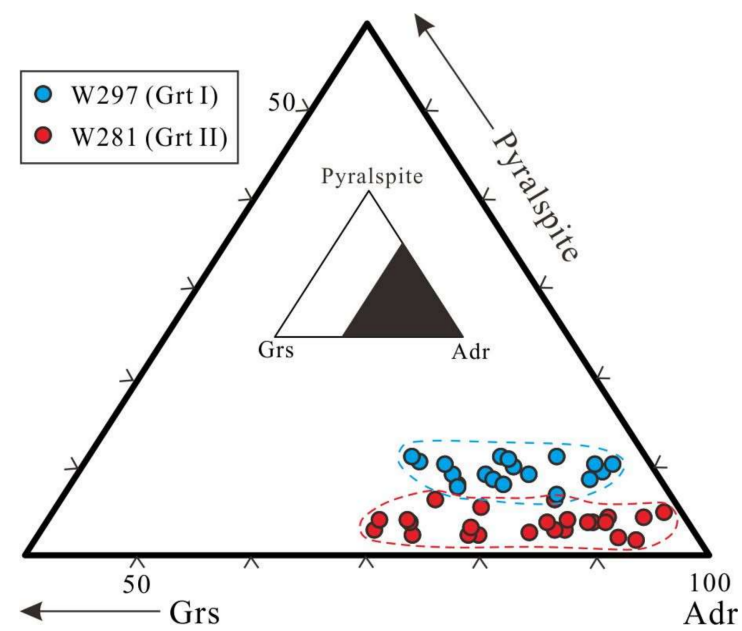

Figure 5. Ternary diagram showing the Beiya garnet compositions.

High U concentrations are found in both Grt I (23.8-64.0 ppm; average: $39.4 \mathrm{ppm})$ and Grt II (2.95-14.3 ppm; average: $8.33 \mathrm{ppm}$ ) (Table 2). Thorium concentrations of Grt I and Grt II range from 0.81 to $3.29 \mathrm{ppm}$ (average: $2.01 \mathrm{ppm}$ ) and $0.89 \mathrm{ppm}$ to $22.1 \mathrm{ppm}$ (average: $6.78 \mathrm{ppm}$ ), with average Th/U ratios of 0.06 and 0.78 , respectively. Chondrite-normalized REE patterns of garnet are shown in Figure 6: All the Beiya U-rich garnet grains are enriched in light REE (LREE) relative to heavy REE (HREE), with total REE ( $\mathrm{REE}$ ) concentrations ranging from 95.8 to $158 \mathrm{ppm}$ (Grt I) and $55.4 \mathrm{ppm}$ to $214 \mathrm{ppm}$ (Grt II). Grt II is characterized by a higher and greater variation of HREE contents (0.61-17.9 ppm) than Grt I (0.47-1.79 ppm). Grt I is characterized by prominent positive Eu anomalies ( $\delta \mathrm{Eu}: 3.5-6.0$; average: 4.1), whereas most Grt II samples exhibit weakly positive/negative or no Eu anomalies ( $\delta \mathrm{Eu}: 0.7-2.4)$.
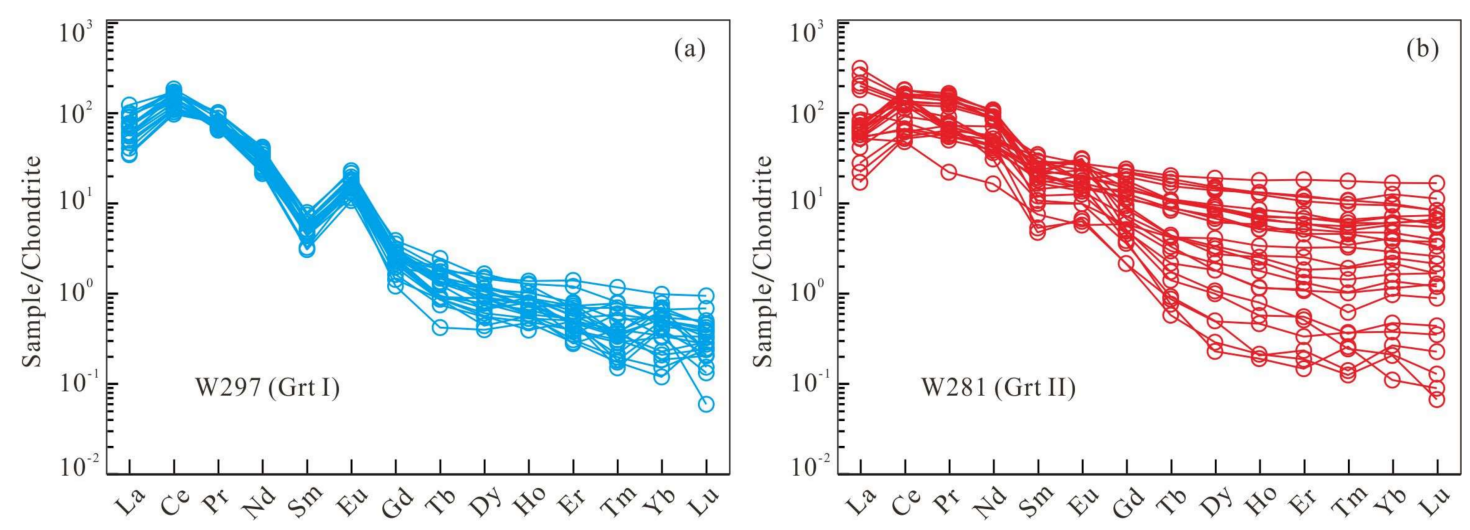

Figure 6. Chondrite-normalized REE patterns of the Beiya garnet, (a) Grt I, (b) Grt II (normalizing values from [54]). 
Table 2. Trae element analysis result (ppm) of the Beiya garnet by laser ablation-inductively coupled plasma-mass spectrometry (LA-ICP-MS).

\begin{tabular}{|c|c|c|c|c|c|c|c|c|c|c|c|c|c|c|c|c|c|c|c|c|c|c|c|}
\hline Point & $\mathrm{Y}$ & La & $\mathrm{Ce}$ & $\operatorname{Pr}$ & Nd & Sm & Eu & Gd & $\mathrm{Tb}$ & Dy & Ho & Er & $\mathrm{Tm}$ & $\mathrm{Yb}$ & Lu & Th & $\mathrm{U}$ & $\Sigma$ REE & LREE & HREE & LREE/HREE $^{1}$ & $\delta E u^{2}$ & $\mathrm{Th} / \mathrm{U}$ \\
\hline \multicolumn{24}{|l|}{ Grt I } \\
\hline W297-3 & 1.35 & 8.37 & 61.8 & 7.36 & 17.1 & 0.95 & 1.05 & 0.57 & 0.06 & 0.23 & 0.04 & 0.08 & 0.00 & 0.07 & 0.01 & 1.90 & 23.8 & 97.6 & 96.6 & 1.05 & 91.7 & 4.0 & 0.08 \\
\hline W297-4 & 1.73 & 13.8 & 92.3 & 9.25 & 18.2 & 0.94 & 1.01 & 0.59 & 0.06 & 0.24 & 0.05 & 0.11 & 0.01 & 0.11 & 0.01 & 2.59 & 25.3 & 137 & 135 & 1.18 & 115 & 3.8 & 0.10 \\
\hline W297-5 & 1.70 & 17.2 & 88.1 & 7.35 & 12.6 & 0.75 & 0.79 & 0.46 & 0.03 & 0.22 & 0.03 & 0.09 & 0.02 & 0.09 & 0.01 & 1.71 & 39.3 & 128 & 127 & 0.95 & 133 & 3.8 & 0.04 \\
\hline W297-6 & 0.83 & 17.1 & 100.3 & 9.46 & 17.2 & 0.72 & 0.98 & 0.44 & 0.03 & 0.15 & 0.03 & 0.08 & 0.01 & 0.03 & 0.01 & 2.48 & 38.3 & 147 & 146 & 0.79 & 185 & 4.9 & 0.06 \\
\hline W297-7 & 0.60 & 17.8 & 82.0 & 6.18 & 10.3 & 0.49 & 0.78 & 0.25 & 0.02 & 0.10 & 0.03 & 0.05 & 0.00 & 0.02 & 0.01 & 0.81 & 45.0 & 118 & 118 & 0.47 & 249 & 6.0 & 0.02 \\
\hline W297-8 & 1.69 & 9.60 & 65.5 & 7.37 & 16.0 & 0.77 & 0.98 & 0.59 & 0.05 & 0.29 & 0.05 & 0.12 & 0.02 & 0.11 & 0.02 & 2.06 & 24.5 & 101 & 100 & 1.25 & 79.9 & 4.3 & 0.08 \\
\hline W297-9 & 1.31 & 13.5 & 78.5 & 7.20 & 14.6 & 0.77 & 0.95 & 0.52 & 0.03 & 0.20 & 0.04 & 0.08 & 0.00 & 0.07 & 0.00 & 1.76 & 32.4 & 116 & 115 & 0.95 & 122 & 4.3 & 0.05 \\
\hline W297-10 & 1.07 & 21.2 & 102 & 8.17 & 14.3 & 0.73 & 0.83 & 0.49 & 0.04 & 0.17 & 0.05 & 0.07 & 0.01 & 0.09 & 0.01 & 1.45 & 38.2 & 148 & 147 & 0.93 & 159 & 4.0 & 0.04 \\
\hline W297-11 & 0.75 & 23.4 & 102 & 6.48 & 9.86 & 0.47 & 0.61 & 0.29 & 0.03 & 0.11 & 0.03 & 0.05 & 0.01 & 0.03 & 0.01 & 1.47 & 51.5 & 143 & 143 & 0.56 & 256 & 4.7 & 0.03 \\
\hline W297-12 & 1.68 & 16.1 & 96.2 & 9.38 & 19.4 & 0.98 & 1.12 & 0.63 & 0.06 & 0.30 & 0.05 & 0.09 & 0.02 & 0.08 & 0.01 & 3.06 & 37.1 & 144 & 143 & 1.23 & 116 & 4.1 & 0.08 \\
\hline W297-13 & 1.96 & 16.6 & 86.5 & 6.88 & 12.6 & 0.79 & 0.85 & 0.49 & 0.07 & 0.28 & 0.04 & 0.13 & 0.01 & 0.13 & 0.00 & 1.66 & 39.9 & 125 & 124 & 1.16 & 107 & 3.9 & 0.04 \\
\hline W297-14 & 1.05 & 17.7 & 80.3 & 6.04 & 10.04 & 0.73 & 0.78 & 0.33 & 0.03 & 0.14 & 0.03 & 0.07 & 0.01 & 0.04 & 0.01 & 0.98 & 49.8 & 116 & 11 & 0.64 & 179 & 4.2 & 0.02 \\
\hline W297-15 & 1.40 & 20.8 & 111 & 9.05 & 14.9 & 0.68 & 0.75 & 0.51 & 0.05 & 0.17 & 0.04 & 0.06 & 0.02 & 0.06 & 0.01 & 2.49 & 46.1 & 158 & 157 & 0.92 & 171 & 3.7 & 0.05 \\
\hline W297-16 & 1.12 & 28.5 & 103 & 7.04 & 11.2 & 0.66 & 0.66 & 0.44 & 0.03 & 0.14 & 0.02 & 0.08 & 0.01 & 0.04 & 0.01 & 1.28 & 64.0 & 152 & 151 & 0.78 & 194 & 3.5 & 0.02 \\
\hline W297-17 & 1.61 & 16.8 & 99.7 & 9.42 & 17.8 & 0.89 & 0.87 & 0.49 & 0.06 & 0.30 & 0.05 & 0.09 & 0.01 & 0.09 & 0.01 & 3.29 & 38.2 & 147 & 146 & 1.10 & 133 & 3.7 & 0.09 \\
\hline W297-18 & 2.36 & 8.11 & 58.5 & 7.32 & 17.9 & 1.20 & 1.24 & 0.64 & 0.06 & 0.42 & 0.07 & 0.11 & 0.02 & 0.12 & 0.01 & 2.81 & 25.5 & 95.8 & 94.3 & 1.46 & 64.7 & 3.9 & 0.11 \\
\hline W297-19 & 1.68 & 11.7 & 67.8 & 7.01 & 14.1 & 0.95 & 1.04 & 0.55 & 0.05 & 0.23 & 0.04 & 0.08 & 0.01 & 0.09 & 0.01 & 2.64 & 39.9 & 104 & 103 & 1.05 & 97.5 & 4.0 & 0.07 \\
\hline W297-20 & 1.26 & 17.8 & 80.6 & 6.36 & 11.7 & 0.71 & 0.82 & 0.40 & 0.06 & 0.20 & 0.03 & 0.06 & 0.01 & 0.06 & 0.00 & 1.81 & 50.9 & 11 & 118 & 0.82 & 144 & 4.3 & 0.04 \\
\hline W297-21 & 2.39 & 10.9 & 66.0 & 7.42 & 18.5 & 1.12 & 1.31 & 0.72 & 0.07 & 0.37 & 0.07 & 0.20 & 0.02 & 0.07 & 0.01 & 2.06 & 26.1 & 10 & 10 & 1.5 & 68.3 & 4.2 & 0.08 \\
\hline W297-22 & 1.31 & 22.4 & 89.4 & 6.67 & 11.6 & 0.86 & 0.77 & 0.40 & 0.03 & 0.23 & 0.03 & 0.05 & 0.01 & 0.06 & 0.01 & 1.31 & 55.0 & 133 & 132 & 0.82 & 161 & 3.5 & 0.02 \\
\hline W297-23 & 1.71 & 12.9 & 71.4 & 6.62 & 13.9 & 0.81 & 0.94 & 0.50 & 0.05 & 0.27 & 0.04 & 0.12 & 0.01 & 0.11 & 0.01 & 1.92 & 36.0 & 108 & 107 & 1.11 & 95.9 & 4.2 & 0.05 \\
\hline W297-24 & 2.93 & 14.3 & 77.0 & 7.14 & 15.4 & 0.99 & 1.13 & 0.79 & 0.09 & 0.38 & 0.08 & 0.23 & 0.03 & 0.17 & 0.02 & 2.33 & 31.0 & 11 & 116 & 1.79 & 64.8 & 3.8 & 0.08 \\
\hline W297-25 & 2.15 & 18.0 & 80.1 & 6.17 & 10.8 & 0.81 & 0.85 & 0.48 & 0.07 & 0.28 & 0.06 & 0.13 & 0.01 & 0.09 & 0.01 & 2.25 & 47.2 & 118 & 117 & 1.14 & 103 & 3.8 & 0.05 \\
\hline \multicolumn{24}{|l|}{ Grt II } \\
\hline W281-1 & 10.7 & 15.3 & 89.3 & 12.7 & 45.7 & 4.35 & 1.57 & 3.60 & 0.40 & 2.27 & 0.41 & 1.11 & 0.16 & 1.19 & 0.18 & 13.7 & 14.3 & 178 & 169 & 9.32 & 18.1 & 1.2 & 0.96 \\
\hline W281-2 & 9.15 & 13.3 & 80.7 & 11.5 & 41.4 & 4.26 & 1.52 & 2.97 & 0.38 & 2.14 & 0.38 & 0.95 & 0.14 & 1.03 & 0.16 & 10.3 & 12.1 & 16 & 15 & 8. & 18.7 & 1.2 & 0.85 \\
\hline W281-3 & 3.33 & 16.8 & 96.4 & 13.9 & 49.3 & 3.91 & 1.72 & 1.90 & 0.16 & 0.69 & 0.14 & 0.30 & 0.05 & 0.37 & 0.04 & 22.1 & 12.0 & 186 & 18 & 3.6 & 49.9 & 1.7 & 1.84 \\
\hline W281-4 & 1.86 & 9.63 & 54.5 & 7.23 & 23.5 & 1.82 & 0.71 & 1.00 & 0.08 & 0.46 & 0.07 & 0.18 & 0.03 & 0.19 & 0.03 & 6.54 & 6.12 & 99.5 & 97.5 & 2.03 & 48.1 & 1.5 & 1.07 \\
\hline W281-6 & 0.31 & 12.1 & 67.0 & 8.35 & 21.6 & 0.80 & 0.36 & 0.44 & 0.02 & 0.07 & 0.01 & 0.03 & 0.00 & 0.03 & 0.00 & 4.80 & 8.19 & 111 & 110 & 0.61 & 179 & 1.7 & 0.59 \\
\hline W281-7 & 0.40 & 14.3 & 95.6 & 13.4 & 38.5 & 1.50 & 0.56 & 0.73 & 0.03 & 0.13 & 0.01 & 0.04 & 0.00 & 0.05 & 0.01 & 8.97 & 9.51 & 165 & 164 & 0.99 & 165 & 1.5 & 0.94 \\
\hline W281-8 & 0.30 & 15.8 & 105 & 15.2 & 47.0 & 2.14 & 0.90 & 0.78 & 0.03 & 0.06 & 0.01 & 0.02 & 0.01 & 0.02 & 0.00 & 13.5 & 10.8 & 187 & 186 & 0.93 & 200 & 1.7 & 1.25 \\
\hline W281-9 & 1.16 & 14.2 & 95.6 & 14.4 & 48.4 & 2.49 & 1.15 & 0.93 & 0.05 & 0.25 & 0.03 & 0.09 & 0.01 & 0.08 & 0.01 & 11.1 & 7.73 & 178 & 176 & 1.45 & 121 & 1.9 & 1.44 \\
\hline W281-10 & 27.0 & 6.47 & 37.0 & 6.71 & 32.2 & 5.17 & 1.55 & 4.82 & 0.74 & 4.72 & 0.99 & 2.94 & 0.44 & 2.81 & 0.41 & 5.69 & 7.72 & 107 & 89.0 & 17.9 & 4.98 & 0.9 & 0.74 \\
\hline W281-11 & 19.5 & 5.14 & 32.6 & 5.97 & 25.7 & 4.20 & 1.33 & 3.96 & 0.57 & 3.45 & 0.74 & 1.97 & 0.27 & 2.10 & 0.28 & 3.56 & 6.34 & 88.3 & 75.0 & 13.3 & 5.62 & 1.0 & 0.56 \\
\hline W281-12 & 11.1 & 3.98 & 31.1 & 5.40 & 22.9 & 3.43 & 1.14 & 2.94 & 0.40 & 2.16 & 0.39 & 1.12 & 0.15 & 1.01 & 0.17 & 3.10 & 5.46 & 76.3 & 68.0 & 8.34 & 8.15 & 1.1 & 0.57 \\
\hline W281-13 & 4.02 & 12.3 & 77.7 & 11.7 & 41.3 & 3.13 & 1.33 & 1.74 & 0.16 & 0.84 & 0.15 & 0.41 & 0.05 & 0.42 & 0.05 & 11.9 & 9.32 & 151 & 147 & 3.82 & 38.6 & 1.6 & 1.28 \\
\hline W281-14 & 0.56 & 19.1 & 77.1 & 6.88 & 14.1 & 0.72 & 0.39 & 0.43 & 0.03 & 0.12 & 0.03 & 0.06 & 0.01 & 0.06 & 0.01 & 3.46 & 7.56 & 119 & 118 & 0.75 & 157 & 2.0 & 0.46 \\
\hline W281-15 & 5.25 & 12.1 & 28.6 & 2.05 & 7.49 & 1.13 & 0.33 & 1.19 & 0.15 & 1.03 & 0.19 & 0.53 & 0.08 & 0.49 & 0.06 & 0.89 & 2.95 & 55.4 & 51.7 & 3.72 & 13.9 & 0.9 & 0.30 \\
\hline
\end{tabular}


Table 2. Cont.

\begin{tabular}{|c|c|c|c|c|c|c|c|c|c|c|c|c|c|c|c|c|c|c|c|c|c|c|c|}
\hline Point & $\mathrm{Y}$ & La & $\mathrm{Ce}$ & Pr & Nd & Sm & Eu & Gd & $\mathrm{Tb}$ & Dy & Ho & Er & $\mathrm{Tm}$ & $\mathrm{Yb}$ & Lu & Th & $\mathbf{U}$ & $\Sigma \mathrm{REE}$ & LREE & HREE & LREE/HREE 1 & $\delta \mathrm{Eu}{ }^{2}$ & $\mathrm{Th} / \mathrm{U}$ \\
\hline \multicolumn{24}{|l|}{ Grt II } \\
\hline W281-18 & 3.75 & 16.4 & 107 & 13.8 & 39.1 & 2.52 & 0.77 & 1.46 & 0.16 & 0.79 & 0.13 & 0.25 & 0.04 & 0.28 & 0.04 & 6.21 & 5.85 & 183 & 180 & 3.14 & 57.3 & 1.1 & 1.06 \\
\hline W281-19 & 12.9 & 23.7 & 46.6 & 5.07 & 19.6 & 3.22 & 0.82 & 2.84 & 0.41 & 2.40 & 0.47 & 1.24 & 0.15 & 1.18 & 0.14 & 1.18 & 5.15 & 108 & 99.0 & 8.84 & 11.2 & 0.8 & 0.23 \\
\hline W281-20 & 11.0 & 12.3 & 40.0 & 5.13 & 20.4 & 3.54 & 1.10 & 3.22 & 0.40 & 2.21 & 0.37 & 0.98 & 0.13 & 0.97 & 0.14 & 3.25 & 5.07 & 90.8 & 82.4 & 8.40 & 9.8 & 1.0 & 0.64 \\
\hline W281-21 & 18.5 & 45.7 & 77.4 & 6.65 & 22.8 & 4.40 & 1.01 & 4.36 & 0.63 & 3.63 & 0.69 & 1.72 & 0.24 & 1.59 & 0.21 & 1.90 & 9.98 & 171 & 158 & 13.1 & 12.1 & 0.7 & 0.19 \\
\hline W281-22 & 9.05 & 12.6 & 38.8 & 4.64 & 17.7 & 2.89 & 0.96 & 2.57 & 0.33 & 1.81 & 0.29 & 0.84 & 0.12 & 0.79 & 0.10 & 2.36 & 4.74 & 84.4 & 77.6 & 6.85 & 11.3 & 1.1 & 0.50 \\
\hline W281-25 & 20.7 & 71.5 & 92.9 & 6.59 & 23.1 & 4.90 & 1.02 & 4.49 & 0.68 & 3.77 & 0.73 & 1.90 & 0.27 & 1.65 & 0.21 & 2.08 & 10.5 & 214 & 200 & 13.7 & 14.6 & 0.7 & 0.20 \\
\hline W281-26 & 2.19 & 14.9 & 74.6 & 10.8 & 39.5 & 3.43 & 1.67 & 1.37 & 0.12 & 0.46 & 0.06 & 0.18 & 0.02 & 0.16 & 0.02 & 10.5 & 7.27 & 147 & 145 & 2.39 & 60.5 & 2.0 & 1.45 \\
\hline W281-27 & 8.75 & 41.5 & 72.0 & 5.81 & 19.8 & 3.26 & 0.89 & 2.23 & 0.33 & 1.70 & 0.29 & 0.74 & 0.11 & 0.64 & 0.09 & 7.12 & 9.68 & 149 & 143 & 6.15 & 23.3 & 1.0 & 0.74 \\
\hline W281-28 & 8.25 & 60.8 & 89.2 & 6.15 & 18.7 & 2.74 & 0.84 & 2.35 & 0.31 & 1.55 & 0.32 & 0.74 & 0.09 & 0.68 & 0.08 & 0.99 & 10.3 & 185 & 178 & 6.13 & 29.1 & 1.0 & 0.10 \\
\hline W281-29 & 0.83 & 18.3 & 92.6 & 12.5 & 45.2 & 3.15 & 1.77 & 1.04 & 0.06 & 0.27 & 0.04 & 0.08 & 0.01 & 0.04 & 0.00 & 13.2 & 9.08 & 175 & 174 & 1.55 & 112 & 2.4 & 1.45 \\
\hline W281-30 & 2.82 & 49.3 & 81.5 & 5.96 & 16.5 & 1.61 & 0.56 & 1.17 & 0.11 & 0.55 & 0.10 & 0.22 & 0.03 & 0.23 & 0.03 & 1.22 & 10.4 & 158 & 155 & 2.43 & 64.0 & 1.2 & 0.12 \\
\hline
\end{tabular}

${ }^{1} \mathrm{LREE}=\mathrm{La}+\mathrm{Ce}+\mathrm{Pr}+\mathrm{Nd}+\mathrm{Sm}+\mathrm{Eu} ; \mathrm{HREE}=\mathrm{Gd}+\mathrm{Tb}+\mathrm{Dy}+\mathrm{Ho}+\mathrm{Er}+\mathrm{Tm}+\mathrm{Yb}+\mathrm{Lu} ;{ }^{2} \cdot \delta \mathrm{Eu}=\left(\mathrm{Eu} / \mathrm{Eu}^{*}\right)_{\mathrm{N}}=\mathrm{Eu}_{\mathrm{N}} /(1 / 2) \mathrm{Sm}_{\mathrm{N}}+(1 / 2) \mathrm{Gd}_{\mathrm{N}}$. 


\subsection{Garnet $U-P b$ Ages}

The U-Pb isotope results of Grt I and Grt II are presented in Table 3. U-Pb ratios vary in the Beiya garnet are generally discordant due to the presence of common $\mathrm{Pb}$. Thus, we take the lower intercept date to represent the garnet age. On Tera-Wasserburg diagrams (Figure 7), the data from Grt I and Grt II define two linear arrays, giving lower intercept ages of $35.8 \pm 0.8 \mathrm{Ma}(\mathrm{MSWD}=0.9, n=20$ ) and $34.0 \pm 2.1 \mathrm{Ma}(\mathrm{MSWD}=1.2, n=16)$, respectively.

Table 3. LA-ICP-MS U-Pb isotope data for garnet from the Beiya deposit.

\begin{tabular}{|c|c|c|c|c|c|c|}
\hline \multirow{2}{*}{ Point } & \multicolumn{2}{|c|}{${ }^{207} \mathrm{~Pb} / 206 \mathrm{~Pb}$} & \multicolumn{2}{|c|}{${ }^{207} \mathrm{~Pb} /{ }^{235} \mathrm{U}$} & \multicolumn{2}{|c|}{${ }^{206} \mathrm{~Pb} /{ }^{238} \mathrm{U}$} \\
\hline & Ratio & 1sigma & Ratio & 1sigma & Ratio & 1sigma \\
\hline \multicolumn{7}{|l|}{ Grt I } \\
\hline W297-1-03 & 0.1078 & 0.0149 & 0.0900 & 0.0092 & 0.0061 & 0.0002 \\
\hline W297-1-04 & 0.1300 & 0.0320 & 0.1100 & 0.0128 & 0.0061 & 0.0003 \\
\hline W297-1-05 & 0.0898 & 0.0147 & 0.0725 & 0.0102 & 0.0059 & 0.0002 \\
\hline W297-1-06 & 0.2506 & 0.0294 & 0.2385 & 0.0267 & 0.0069 & 0.0002 \\
\hline W297-1-07 & 0.1167 & 0.0261 & 0.0969 & 0.0110 & 0.0060 & 0.0003 \\
\hline W297-1-09 & 0.0931 & 0.0215 & 0.0765 & 0.0075 & 0.0060 & 0.0002 \\
\hline W297-1-10 & 0.0973 & 0.0206 & 0.0838 & 0.0090 & 0.0062 & 0.0002 \\
\hline W297-1-11 & 0.1006 & 0.0260 & 0.0800 & 0.0087 & 0.0058 & 0.0002 \\
\hline W297-1-12 & 0.1081 & 0.0196 & 0.0880 & 0.0082 & 0.0059 & 0.0002 \\
\hline W297-1-14 & 0.2618 & 0.0338 & 0.2555 & 0.0166 & 0.0071 & 0.0003 \\
\hline W297-1-15 & 0.2181 & 0.0372 & 0.2062 & 0.0213 & 0.0069 & 0.0004 \\
\hline W297-1-17 & 0.2804 & 0.0417 & 0.3148 & 0.0260 & 0.0081 & 0.0003 \\
\hline W297-1-20 & 0.1042 & 0.0201 & 0.0879 & 0.0086 & 0.0061 & 0.0002 \\
\hline W297-1-21 & 0.1747 & 0.0347 & 0.1617 & 0.0162 & 0.0067 & 0.0003 \\
\hline W297-1-25 & 0.0889 & 0.0169 & 0.0758 & 0.0105 & 0.0062 & 0.0002 \\
\hline W297-1-26 & 0.1029 & 0.0145 & 0.0899 & 0.0083 & 0.0063 & 0.0002 \\
\hline W297-1-27 & 0.6246 & 0.0376 & 1.7264 & 0.1087 & 0.0200 & 0.0010 \\
\hline W297-1-28 & 0.1993 & 0.0226 & 0.1787 & 0.0258 & 0.0065 & 0.0004 \\
\hline W297-1-30 & 0.4950 & 0.0379 & 0.9488 & 0.0404 & 0.0139 & 0.0004 \\
\hline W297-1-31 & 0.4569 & 0.0366 & 0.7666 & 0.0457 & 0.0122 & 0.0005 \\
\hline \multicolumn{7}{|l|}{ Grt II } \\
\hline W281-1-01 & 0.2996 & 0.0477 & 0.3466 & 0.0393 & 0.0084 & 0.0004 \\
\hline W281-1-04 & 0.4768 & 0.0564 & 0.8491 & 0.0814 & 0.0129 & 0.0007 \\
\hline W281-1-08 & 0.7728 & 0.0410 & 6.4467 & 0.2126 & 0.0605 & 0.0016 \\
\hline W281-1-09 & 0.7856 & 0.0415 & 7.1790 & 0.4840 & 0.0663 & 0.0040 \\
\hline W281-1-12 & 0.6480 & 0.0563 & 2.5820 & 0.2228 & 0.0289 & 0.0020 \\
\hline W281-1-13 & 0.7230 & 0.0618 & 2.8244 & 0.1252 & 0.0283 & 0.0010 \\
\hline W281-1-14 & 0.1749 & 0.0372 & 0.1563 & 0.0318 & 0.0065 & 0.0004 \\
\hline W281-1-15 & 0.3994 & 0.0484 & 0.5604 & 0.0444 & 0.0102 & 0.0005 \\
\hline W281-1-18 & 0.5441 & 0.0541 & 1.1597 & 0.0768 & 0.0155 & 0.0008 \\
\hline W281-1-21 & 0.7054 & 0.0631 & 3.4552 & 0.2264 & 0.0355 & 0.0019 \\
\hline W281-1-23 & 0.7634 & 0.0560 & 6.2413 & 0.3263 & 0.0593 & 0.0026 \\
\hline W281-1-24 & 0.1625 & 0.0217 & 0.1367 & 0.0202 & 0.0061 & 0.0003 \\
\hline W281-1-25 & 0.6523 & 0.0392 & 1.4804 & 0.2499 & 0.0165 & 0.0019 \\
\hline W281-1-26 & 0.5611 & 0.0586 & 1.0598 & 0.0635 & 0.0137 & 0.0006 \\
\hline W281-1-27 & 0.1607 & 0.0234 & 0.1272 & 0.0306 & 0.0057 & 0.0004 \\
\hline W281-1-29 & 0.6043 & 0.0660 & 1.1606 & 0.0680 & 0.0139 & 0.0007 \\
\hline
\end{tabular}



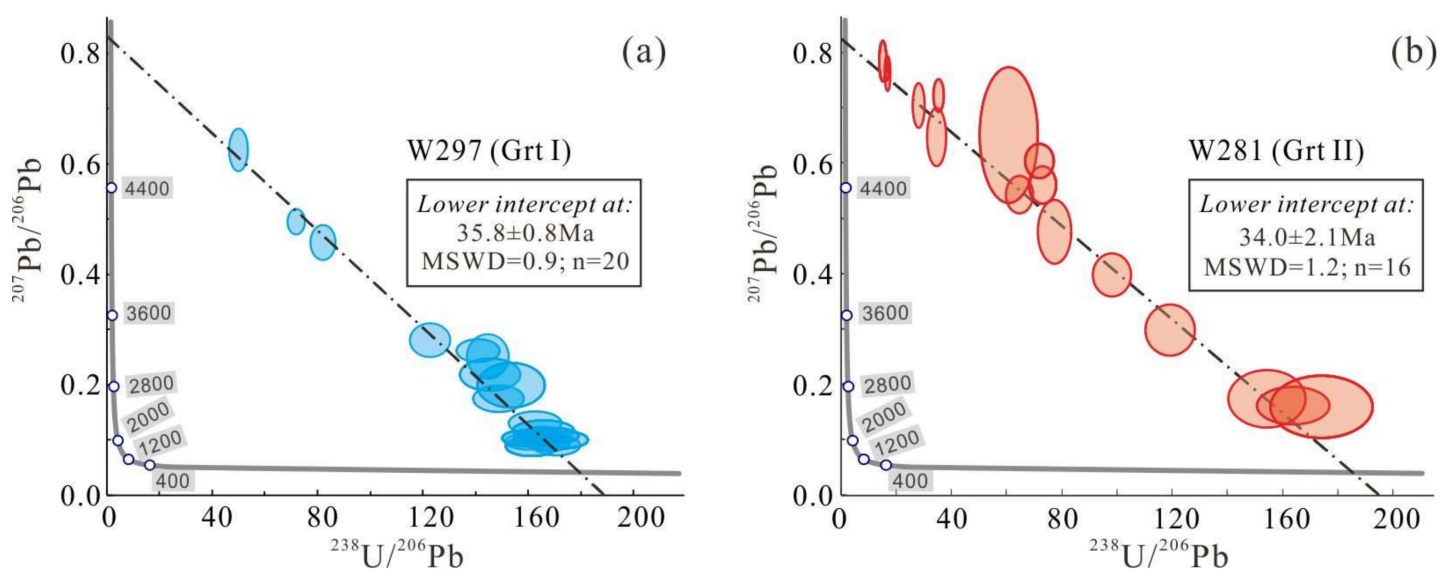

Figure 7. Tera-Wasserburg diagram for the Beiya garnet: (a) W281, (b) W297.

\section{Discussion}

\subsection{Geochronology of the Beiya Garnet}

In this study, lower intercept ages in the Tera-Wasserburg diagram are taken to represent the crystallization age of garnet [55]. It is not only because the Beiya garnet have large initial common $\mathrm{Pb}$ contents, but also because this approach has been proven to be more suitable for young ( 35- 3 Ma) garnet $[55,56]$. The larger analytical uncertainty associated with the U-Pb age for Grt II (34.0 $\pm 2.1 \mathrm{Ma})$ most likely results from the lower $\mathrm{U}$ and higher common Pb contents than Grt I (35.8 $\pm 0.8 \mathrm{Ma})$ (Table 2; Figure 7), since the calculated uncertainty is inversely correlated with both of the latter features. In general, matrix-matched reference materials are needed for LA-ICP-MS U-Pb analysis, however, to date the availability of a high-quality garnet standard for LA-ICP-MS dating analysis is lacking. The garnet standard Willsboro Andradite [55] ( 1020 Ma, Adirondacks, USA), which contains almost exclusively radiogenic $\mathrm{Pb}$ and yields a mean ${ }^{206} \mathrm{~Pb} /{ }^{238} \mathrm{U}$ age of $1022 \pm 16 \mathrm{Ma}$ (ID-TIMS) is useful. However, the Willsboro Andradite has low U content (approximate $1 \mathrm{ppm}$ ) and hence yields large uncertainties associated with the isotope ratio measurements, which makes it an unsuitable reference material for this study. Previous studies have shown that accurate and precise garnet U-Pb ages can be obtained by using larger laser spot sizes and non-matrix matched external standards, such as zircons (91500 and GJ-1) [14,56]. Therefore, the non-matrix matched external zircon standard 91500 was used in this study.

Paragenetic evidence indicates that the Beiya garnet was formed during the prograde skarn stage. Our age data reported here indicate that the Beiya skarn alteration initiated at around 35.8 Ma. Previous studies suggested that the Beiya ore-related porphyry were emplaced at $36.1 \pm 0.4 \mathrm{Ma}$ [23], and that the Beiya retrograde skarn alteration occurred at 33.1 $\pm 1.0 \mathrm{Ma}$ based on titanite U-Pb ages [48]. Given the uncertainties associated with the age determinations, the Grt I date overlaps with that of the zircon age for the porphyry, and the Grt II date is within uncertainty of the titanite date for the retrograde alteration. The garnet data is consistent with previous studies given the associated uncertainties, which reflects the reliability of the garnet $\mathrm{U}-\mathrm{Pb}$ dating technique used in this study, and provides an alternative choice when studying the geochronology of skarns.

Beiya has been studied for many years, and its geology, geochemistry and geochronology are well established, which benefit the new garnet chronometer test and application. The important implications for garnet $\mathrm{U}-\mathrm{Pb}$ dating are: (1) for some distal skarn alteration such as the skarn $\mathrm{Pb}-\mathrm{Zn}$ deposit, the minimum age of mineralization is difficult be constrain by the correlated intrusions since most ore-related intrusions are distant from the mineralization zones; (2) for most skarn deposits that have clear petrogenetic relationship with their associated intrusions, their ages are usually constrained by the intrusion age (e.g., zircon $\mathrm{U}-\mathrm{Pb}$ ). However, it is well established that garnet crystallization 
occurs during or slightly after magma emplacement. Therefore, the garnet U-Pb dating, if possible, may provide a more precise minimum age for either the skarn alteration or the mineralization ages.

\subsection{Metallogenic Implications}

Previous studies suggest that the incorporation of $\mathrm{U}, \mathrm{REEs}$ and $\mathrm{Y}$ into garnet is mainly controlled by crystal chemistry, which is possible by replacement of $\mathrm{X}^{2+}$ cations such as $\mathrm{Ca}^{2+}$ in the dodecahedral position on the basis of ionic radius (i.e., Equations (1) and (2)) [5,19]:

$$
\begin{gathered}
\mathrm{Ca}_{3}(\mathrm{Al}, \mathrm{Fe})_{2} \mathrm{Si}_{3} \mathrm{O}_{12}+3 \mathrm{Eu}^{2+}{ }_{(\mathrm{aq})}=\mathrm{Eu}_{3}(\mathrm{Al}, \mathrm{Fe})_{2} \mathrm{Si}_{3} \mathrm{O}_{12}+3 \mathrm{Ca}^{2+}{ }_{(\mathrm{aq})} \\
\mathrm{Ca}_{3}(\mathrm{Al}, \mathrm{Fe})_{2} \mathrm{Si}_{3} \mathrm{O}_{12}+3 \mathrm{REE}^{2+}{ }_{(\mathrm{aq})}+3\left(\mathrm{Al}^{3+}{ }^{{ }_{\mathrm{Fe}}}{ }^{3+}\right)_{(\mathrm{aq})}=\mathrm{REE}_{3}(\mathrm{Al}, \mathrm{Fe})_{2}(\mathrm{Al}, \mathrm{Fe})_{3} \mathrm{O}_{12}+3 \mathrm{Ca}^{2+}{ }_{(\mathrm{aq})}+3 \mathrm{Si}^{4+}{ }_{(\mathrm{aq})}
\end{gathered}
$$

However, increasing evidences show that the incorporation of $Y$, REEs and $U$ into garnet is not only controlled by its crystal chemistry, but also affected by the external factors such as fluid composition, $\mathrm{W} / \mathrm{R}$ ratios, physicochemical conditions, mineral growth kinetics and metasomatism dynamics [5,16,57]. In this study, positive correlations are found between $U$ and LREE contents (Figure 8a), implying that the incorporation of $\mathrm{U}$ into Beiya garnet is possibly controlled by a substitution mechanism [14]. The same correlation between $U$ and $\Sigma$ REEs (Figure $8 b$ ) probably results from the strong enrichment in LREEs relative to HREEs because no positive correlation could be found between $U$ and HREE contents (Figure 8c). However, there is no linear relationships between $Y$ and REE concentrations, REE and $\mathrm{Al}$ concentrations, REE and Ca concentrations, as well as REEs and $\mathrm{Fe}^{3+}$ concentrations in both Grt I and Grt II (Figure 8d-g). This implies that the incorporation of those trace elements into the Beiya U-rich garnet was not governed solely by isovalent and coupled substitution mechanisms, but may be strongly governed by fluid and rock chemistry (surface adsorption and occlusion) [58].

The Beiya ore-forming fluids were suggested to be magma-derived [42], and the REE patterns of Grt I and Grt II are similar to those of the magmatic-hydrothermal fluids, as featured by the LREE-enrichments, HREE-depletions and varying positive Eu anomalies (Figure 6). Such phenomena corroborate the occurrence of infiltration metasomatism, in which the fluid-dominant system can significantly change the REE content of the rocks [59], and the REE patterns of garnet could follow the pattern of magmatic-hydrothermal fluids. Similar REE pattern of garnet could be found in andradite-rich garnet from the skarn Fe deposit in North China Craton [14]. Therefore, we propose that the Beiya high-U garnet was likely formed via infiltration metasomatism, which is commonly associated with Fe-rich minerals. During the infiltration metasomatism, the Beiya garnets may have grown rapidly in magmatic-derived fluids under high water/rock ratio conditions, therefore the adsorption probably has a major control on the REE patterns of the Beiya garnet. These features (the Fe-rich garnet commonly grow rapidly by infiltration metasomatism with high $\mathrm{W} / \mathrm{R}$ ratios in magmatic-derived fluids) of Beiya garnet are similar to the garnet from the Crown Jewel gold deposit, one of the largest Au skarn deposits in North America [5,60].

It is suggested that $\mathrm{pH}$ has a major effect on REE fractionation [59]; under mildly acidic conditions, chlorine in hydrothermal fluids would enhance the stability of soluble $\mathrm{Eu}^{2+}$, resulting in positive $\mathrm{Eu}$ anomalies [17,61]; whereas under near neutral conditions the fluids are HREE-enriched and LREE-depleted with negative or no Eu anomaly [16,59]. At Beiya, Grt I exhibits clear positive Eu anomalies, but Grt II shows weak or no Eu anomalies with smaller LREE/HREE fractionation than Grt I, which indicate that Grt I and Grt II may have formed under a mildly acidic and near neutral condition, respectively. $U$ content in garnet can be used to determine the redox conditions of hydrothermal fluid systems $[5,16,17,19]$. Based on ionic radius, $\mathrm{U}^{4+}$ is much more likely to substitute into garnet than $\mathrm{U}^{6+}$ [19]. Therefore, the lower concentration of $\mathrm{U}$ and higher Th/U ratio in Grt II indicates a more oxidizing (higher $f \mathrm{O}_{2}$ ) condition than that of Grt I (Figure $8 \mathrm{~h}$ ).

At Beiya, paragenetic observations and U-Pb dating reported in this study suggest that Grt I (relatively Fe-low) was formed slightly earlier than Grt II (relatively Fe-rich), which is consistent with 
most of the Cu-Fe skarn-related garnets worldwide [5]. The physicochemical ore-forming condition changes we proposed here could have major influence on the incorporation of trace elements into the garnet crystal lattice [16]. Based on the U contents, REE patterns and Eu anomalies of Grt I and Grt II (Figure $8 \mathrm{~h}$ ), the hydrothermal fluid evolution in the Beiya prograde skarn alteration stage could be summarized as a transition from a relatively reduced and acidic fluid system to a relatively oxidized and neutral one.
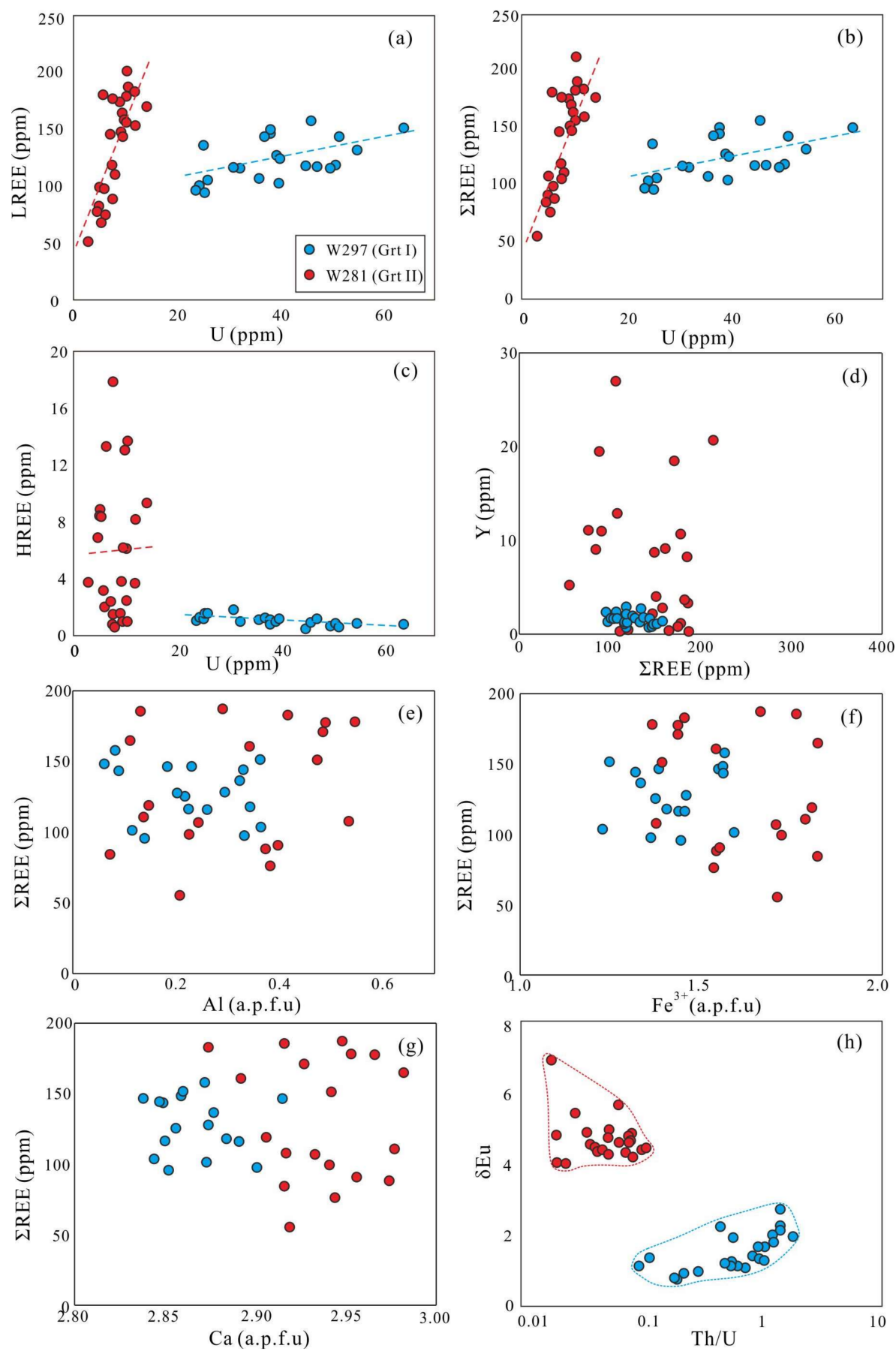

Figure 8. Diagram of (a) U vs. LREE, (b) U vs. HREE, (c) U vs. RREE, (d) $\Sigma$ REE vs. $\mathrm{Y}$, (e-g) $\mathrm{Al}, \mathrm{Fe}^{3+}$ and Ca vs. $\Sigma$ REE and (h) Th/U vs. $\delta$ Eu for the Beiya garnet. 


\section{Conclusions}

(1) Two types of U-rich garnet are distinguished from the Beiya skarn ores: Grt I and Grt II. Both Grt I and Grt II are Fe-rich grandites, although Grt II is richer in Fe than Grt I. Grt I and Grt II yield lower intercept $\mathrm{U}-\mathrm{Pb}$ ages of $35.8 \pm 0.8 \mathrm{Ma}$ and $34.0 \pm 2.1 \mathrm{Ma}$, respectively, which is consistent with previous studies within uncertainty and provides a reliable alternative in dating skarn alteration/mineralization.

(2) Trace element characteristics of Grt I and Grt II suggest that from the early to late prograde skarn, the ore-forming fluids may have changed from being relatively reduced and acidic to relatively oxidized and neutral.

Acknowledgments: This work was jointly funded by the National Natural Science Foundation of China (41602067, U1302233, 41702067), China Postdoctoral Science Foundation (2015M582457), National Key Basic Research Program (2015CB452604; PRC Ministry of Science and Technology), Natural Science Foundation of Guangdong Province (2017A030313246), and the Project Supported by Guangdong Province Universities and Colleges Pearl River Scholar Funded Scheme (2011). We thank Jianping Liu and Yuzhou Feng for helping with the EPMA analysis, and Rongqing Zhang and Yu Zhang for advising on the LA-ICP-MS analysis. Wenchang Li and Zhangrong Liu are thanked for the fieldwork and sampling support. Two anonymous reviewers are thanked for constructive reviews that greatly improved the quality of this paper.

Author Contributions: Yu Fu and Xiaoming Sun conceived and designed the experiments; Yu Fu and Dengfeng Li performed the experiments; Dengfeng Li and Hai Lin analyzed the data; Yu Fu wrote the paper, assisted by all other authors.

Conflicts of Interest: The authors declare no conflict of interest.

\section{References}

1. Baghban, S.; Hosseinzadeh, M.R.; Moayyed, M.; Mokhtari, M.A.A.; Gregory, D.D.; Nia, H.M. Chemical composition and evolution of the garnets in the Astamal Fe-LREE distal skarn deposit, Qara-Dagh-Sabalan metallogenic belt, Lesser Caucasus, NW Iran. Ore Geol. Rev. 2016, 78, 166-175. [CrossRef]

2. Caddick, M.J.; Konopásek, J.; Thompson, A.B. Preservation of garnet growth zoning and the duration of prograde metamorphism. J. Petrol. 2010, 51, 2327-2347. [CrossRef]

3. Chen, Y.X.; Zhou, K.; Zheng, Y.F.; Chen, R.X.; Hu, Z. Garnet geochemistry records the action of metamorphic fluids in ultrahigh-pressure dioritic gneiss from the Sulu orogen. Chem. Geol. 2015, 398, 46-60. [CrossRef]

4. Crowe, D.E.; Riciputi, L.R.; Bezenek, S.; Ignatiev, A. Oxygen isotope and trace element zoning in hydrothermal garnets: Windows into large-scale fluid-flow behavior. Geology 2001, 29, 479-482. [CrossRef]

5. Gaspar, M.; Knaack, C.; Meinert, L.D.; Moretti, R. REE in skarn systems: A LA-ICP-MS study of garnets from the Crown Jewel gold deposit. Geochim. Cosmochim. Acta 2008, 72, 185-205. [CrossRef]

6. Jamtveit, B.; Wogelius, R.A.; Fraser, D.G. Zonation patterns of skarn garnets: Records of hydrothermal system evolution. Geology 1993, 21, 113-116. [CrossRef]

7. Raimondo, T.; Payne, J.; Wade, B.; Lanari, P.; Clark, C.; Hand, M. Trace element mapping by LA-ICP-MS: Assessing geochemical mobility in garnet. Contrib. Mineral. Petrol. 2017, 172, 17. [CrossRef]

8. Jung, S.; Mezger, K. U-Pb garnet chronometry in high-grade rocks-Case studies from the central Damara orogen (Namibia) and implications for the interpretation of Sm-Nd garnet ages and the role of high U-Th inclusions. Contrib. Mineral. Petrol. 2003, 146, 382-396. [CrossRef]

9. Schmidt, A.; Pourteau, A.; Candan, O.; Oberhänsli, R. Lu-Hf geochronology on cm-sized garnets using microsampling: New constraints on garnet growth rates and duration of metamorphism during continental collision (Menderes Massif, Turkey). Earth Planet. Sci. Lett. 2015, 432, 24-35. [CrossRef]

10. Scherer, E.E.; Cameron, K.L.; Blichert-Toft, J. Lu-Hf garnet geochronology: Closure temperature relative to the Sm-Nd system and the effects of trace mineral inclusions. Geochim. Cosmochim. Acta 2000, 64, 3413-3432. [CrossRef]

11. Dewolf, C.P.; Zeissler, C.J.; Halliday, A.N.; Mezger, K.; Essene, E.J. The role of inclusions in U-Pb, and Sm-Nd garnet geochronology: Stepwise dissolution experiments and trace uranium mapping by fission track analysis. Geochim. Cosmochim. Acta 1996, 60, 121-134. [CrossRef] 
12. Mezger, K.; Hanson, G.N.; Bohlen, S.R. U-Pb systematics of garnet: Dating the growth of garnet in the Late Archean Pikwitonei granulite domain at Cauchon and Natawahunan Lakes, Manitoba, Canada. Contrib. Mineral. Petrol. 1989, 101, 136-148. [CrossRef]

13. Mezger, K.; Rawnsley, C.M.; Bohlen, S.R.; Hanson, G.N. U-Pb garnet, sphene, monazite, and rutile ages: implications for the duration of high-grade metamorphism and cooling histories, Adirondack Mts., New York. J. Geol. 1991, 99, 415-428. [CrossRef]

14. Deng, X.D.; Li, J.W.; Luo, T.; Qiang, H. Dating magmatic and hydrothermal processes using andradite-rich garnet U-Pb geochronometry. Contrib. Mineral. Petrol. 2017, 172, 71. [CrossRef]

15. Zhang, Y.; Shao, Y.; Zhang, R.; Li, D.; Liu, Z.; Chen, H. Dating ore deposit using garnet U-Pb geochronology: Example from the Xinqiao Cu-S -Fe-Au deposit, Eastern China. Minerals 2018, 8, 31. [CrossRef]

16. Zhai, D.G.; Liu, J.J.; Zhang, H.Y.; Wang, J.P.; Su, L.; Yang, X.A.; Wu, S.H. Origin of oscillatory zoned garnets from the Xieertala Fe-Zn skarn deposit, northern China: In situ LA-ICP-MS evidence. Lithos 2014, 190-191, 279-291. [CrossRef]

17. Zhang, Y.; Shao, Y.J.; Wu, C.D.; Chen, H.Y. LA-ICP-MS trace element geochemistry of garnets: Constraints on hydrothermal fluid evolution and genesis of the Xinqiao $\mathrm{Cu}-\mathrm{S}-\mathrm{Fe}-\mathrm{Au}$ deposit, eastern China. Ore Geol. Rev. 2017, 86, 426-439. [CrossRef]

18. Park, C.; Song, Y.; Kang, I.M.; Shim, J.; Chung, D.; Park, C.S. Metasomatic changes during periodic fluid flux recorded in grandite garnet from the Weondong W-skarn deposit, South Korea. Chem. Geol. 2017, 451, 135-153. [CrossRef]

19. Smith, M.P.; Henderson, P.; Jeffries, T.E.; Long, J.; Williams, C. The Rare Earth Elements and uranium in garnets from the Beinn an Dubhaich Aureole, Skye, Scotland, UK: Constraints on processes in a dynamic hydrothermal system. J. Petrol. 2004, 45, 457-484. [CrossRef]

20. Deng, J.; Wang, Q.; Li, G.; Santosh, M. Cenozoic tectono-magmatic and metallogenic processes in the Sanjiang region, southwestern China. Earth-Sci. Rev. 2014, 138, 268-299. [CrossRef]

21. Deng, X.D.; Li, J.W.; Zhou, M.F.; Zhao, X.F.; Yan, D.R. In-situ LA-ICPMS trace elements and U-Pb analysis of titanite from the Mesozoic Ruanjiawan W-Cu-Mo skarn deposit, Daye district, China. Ore Geol. Rev. 2015, 65, 990-1004. [CrossRef]

22. Hou, Z.; Zeng, P.; Gao, Y.; Du, A.; Fu, D. Himalayan Cu-Mo-Au mineralization in the eastern Indo-Asian collision zone: Constraints from Re-Os dating of molybdenite. Miner. Depos. 2006, 41, 33-45. [CrossRef]

23. Fu, Y.; Sun, X.; Lin, H.; Zhou, H.; Li, X.; Ouyang, X.; Jiang, L.; Shi, G.; Liang, Y. Geochronology of the giant Beiya gold-polymetallic deposit in Yunnan Province, Southwest China and its relationship with the petrogenesis of alkaline porphyry. Ore Geol. Rev. 2015, 71, 138-149. [CrossRef]

24. Deng, J.; Wang, Q.; Li, G.; Hou, Z.; Jiang, C.; Danyushevsky, L. Geology and genesis of the giant Beiya porphyry-skarn gold deposit, northwestern Yangtze Block, China. Ore Geol. Rev. 2015, 70, 457-485. [CrossRef]

25. Liu, B.; Liu, H.; Zhang, C.; Mao, Z.; Zhou, Y.; Huang, H.; He, Z.; Su, G. Geochemistry and geochronology of porphyries from the Beiya gold-polymetallic orefield, western Yunnan, China. Ore Geol. Rev. 2015, 69, 360-379. [CrossRef]

26. Fu, Y.; Sun, X.; Zhou, H.; Lin, H.; Jiang, L.; Yang, T. In-situ LA-ICP-MS trace elements analysis of scheelites from the giant Beiya gold-polymetallic deposit in Yunnan Province, Southwest China and its metallogenic implications. Ore Geol. Rev. 2017, 80, 828-837. [CrossRef]

27. He, W.; Mo, X.; He, Z.; White, N.C.; Chen, J.; Yang, K.; Wang, R.; Yu, X.; Dong, G.; Huang, F. The geology and mineralogy of the Beiya skarn gold deposit in Yunnan, Southwest China. Econ. Geol. 2015, 110, 1625-1641. [CrossRef]

28. Meinert, L.; Hedenquist, J.; Satoh, H.; Matsuhisa, Y. Formation of Anhydrous and Hydrous Skarn in Cu-Au Ore Deposits by Magmatic Fluids. Econ. Geol. 2003, 98, 147-156. [CrossRef]

29. Li, D.; Fu, Y.; Sun, X.; Hollings, P.; Liao, J.; Liu, Q.; Feng, Y.; Liu, Y.; Lai, C. LA-ICP-MS trace element mapping: Element mobility of hydrothermal magnetite from the giant Beiya Fe-Au skarn deposit, SW China. Ore Geol. Rev. 2018, 92, 463-474. [CrossRef]

30. He, Z.H.; Guan, D.R.; He, W.Y.; Zhou, Y.M.; Fu, D.G.; Yang, S.; Wang, L.; Li, W.H.; Su, G.S.; Yang, R. Exploration moldel of Beiya superlarge gold-polymetallic deposit, northwestern Yunnan. Miner. Depos. 2016, 35, 261-282.

31. Mao, J.; Zhou, Y.; Liu, H.; Zhang, C.; Fu, D.; Liu, B. Metallogenic setting and ore genetic model for the Beiya porphyry-skarn polymetallic Au orefield, western Yunnan, China. Ore Geol. Rev. 2017, 86, 21-34. [CrossRef] 
32. Mao, J.; Pirajno, F.; Lehmann, B.; Luo, M.; Berzina, A. Distribution of porphyry deposits in the Eurasian continent and their corresponding tectonic settings. J. Asian Earth Sci. 2014, 79, 576-584. [CrossRef]

33. Wang, J.; Yin, A.; Harrison, T.M.; Grove, M.; Zhang, Y.; Xie, G. A tectonic model for Cenozoic igneous activities in the eastern Indo-Asian collision zone. Earth Planet. Sci. Lett. 2001, 188, 123-133. [CrossRef]

34. Hou, Z.; Zhong, D.; Deng, W. A tectonic model for porphyry copper-molybdenum-gold metallogenic belts on the eastern margin of the Qinghai-Tibet Plateau. Geol. China 2004, 31, 1-14.

35. He, W.Y.; Mo, X.X.; Yang, L.Q.; Xing, Y.L.; Dong, G.C.; Yang, Z.; Gao, X.; Bao, X.S. Origin of the Eocene porphyries and mafic microgranular enclaves from the Beiya porphyry Au polymetallic deposit, western Yunnan, China: Implications for magma mixing/mingling and mineralization. Gondwana Res. 2016, 40, 230-248. [CrossRef]

36. Guo, X.; Wang, Z.; Chen, X.; Wang, X.; Wang, S. Machangqing porphyry Cu-Mo-Au deposit, Yunnan Province: Geological characteristics and its genesis. Acta Geol. Sin. 2009, 83, 1901-1914.

37. Lu, Y.; Kerrich, R.; Kemp, A.I.S.; McCuaig, T.C.; Hou, Z.; Hart, C.J.R.; Li, Z.; Cawood, P.A.; Bagas, L.; Yang, Z.; et al. Intracontinental eocene-oligocene porphyry Cu mineral systems of Yunnan, Western Yangtze Craton, China: Compositional characteristics, sources, and implications for continental collision metallogeny. Econ. Geol. 2013, 108, 1541-1576. [CrossRef]

38. Xu, X.; Cai, X.; Song, B.; Zhang, B.; Ying, H.; Xiao, Q.; Wang, J. Petrologic, chronological and geochemistry characteristics and formation mechanism of alkaline porphyries in the Beiya gold district, western Yunnan. Acta Petrol. Sin. 2006, 22, 631-642.

39. Xue, C.; Hou, Z.; Liu, X.; Yang, Z.; Liu, Y.; Hao, B. Petrogenesis and metallogenesis of the Beiya gold-polymetallic ore district, northwestern Yunnan province, China: Responses to the Indo-Asian collisional processes. Acta Petrol. Sin. 2008, 24, 457-472.

40. Xu, X.; Cai, X.; Xiao, Q.; Peters, S.G. Porphyry Cu-Au and associated polymetallic Fe-Cu-Au deposits in the Beiya Area, western Yunnan Province, south China. Ore Geol. Rev. 2007, 31, 224-246. [CrossRef]

41. Li, W.; Wang, J.; He, Z.; Dou, S. Formation of Au-polymetallic ore deposits in alkaline porphyries at Beiya, Yunnan, Southwest China. Ore Geol. Rev. 2016, 73, 241-252. [CrossRef]

42. He, W.Y. The Beiya Giant Gold-Polymetallic Deposit: Magmatism and Metallogenic Model; China University of Geosciences: Beijing, China, 2014.

43. Lu, Y.; Kerrich, R.; Cawood, P.A.; McCuaig, T.C.; Hart, C.J.R.; Li, Z.; Hou, Z.; Bagas, L. Zircon SHRIMP U-Pb geochronology of potassic felsic intrusions in western Yunnan, SW China: Constraints on the relationship of magmatism to the Jinsha suture. Gondwana Res. 2012, 22, 737-747. [CrossRef]

44. Xu, S. Metallogenic Modeling of the Beiya Gold Deposit in Western Yunnan and Its Relation to the Cenozoic Alkali-Rich Porphries; China University of Geosciences: Beijing, China, 2007.

45. He, W.; Mo, X.; Yu, X.; He, Z.; Dong, G.; Liu, X.; Su, G.; Huang, X. Zircon U-Pb and molybdenite Re-Os dating for the Beiya gold-polymetallic deposit in the western Yunnan Province and its geological significance. Acta Petrol. Sin. 2013, 29, 1301-1310.

46. Jiang, C.; Wang, Q.; Li, G.; Ma, N.; Hu, Z. Relative oxidation states of intrusions in Beiya gold-polymetallic deposit in Sanjiang area, Yunnan, SW China. Acta Geol. Sin. 2013, 29, 3925-3936.

47. He, W.; Yu, X.; Mo, X.; He, Z.; Li, Y.; Huang, X.; Su, G. Genetic type and the relationship between alkali-rich intrusion and mineralization of Beiya gold-polymetallic ore field, western Yunnan Province, China. Acta Geol. Sin. 2012, 28, 1401-1421.

48. Fu, Y.; Sun, X.; Zhou, H.; Lin, H.; Yang, T. In-situ LA-ICP-MS U-Pb geochronology and trace elements analysis of polygenetic titanite from the giant Beiya gold-polymetallic deposit in Yunnan Province, Southwest China. Ore Geol. Rev. 2016, 77, 43-56. [CrossRef]

49. Pearce, N.J.G.; Perkins, W.T.; Westgate, J.A.; Gorton, M.P.; Jackson, S.E.; Neal, C.R.; Chenery, S.P. A compilation of new and published major and trace element data for NIST SRM 610 and NIST SRM 612 glass reference materials. Geostand. Geoanal. Res. 1997, 21, 115-144. [CrossRef]

50. Wiedenbeck, M.; AllÉ, P.; Corfu, F.; Griffin, W.L.; Meier, M.; Oberli, F.; Quadt, A.V.; Roddick, J.C.; Spiegel, W. Three natural zircon standards for U-Th-Pb, Lu-Hf, trace element and REE analyses. Geostand. Geoanal. Res. 1995, 19, 1-23. [CrossRef]

51. Slama, J.; Kosler, J.; Condon, D.J.; Crowley, J.L.; Gerdes, A.; Hanchar, J.M.; Horstwood, M.S.A.; Morris, G.A.; Nasdala, L.; Norberg, N.; et al. Plĕsovice zircon-A new natural reference material for U-Pb and Hf isotopic microanalysis. Chem. Geol. 2008, 249, 1-35. [CrossRef] 
52. Liu, Y.; Gao, S.; Hu, Z.; Gao, C.; Zong, K.; Wang, D. Continental and oceanic crust recycling-induced melt-peridotite interactions in the Trans-North China Orogen: U-Pb dating, Hf isotopes and trace elements in zircons from mantle xenoliths. J. Petrol. 2010, 51, 537-571. [CrossRef]

53. Ludwig, K.R. User's Manual for Isoplot 3.0: A Geochronological Toolkit for Microsoft Excel; Berkeley Geochronology Center: Berkeley, CA, USA, 2003.

54. Sun, S.S.; McDonough, W.F. Chemical and isotopic systematics of oceanic basalts: Implications for mantle composition and processes. In Magmatism in the Ocean Basins; Snder, A.D., Norry, M.J., Eds.; Geological Society Special Publication: London, UK, 1989; Volume 42, pp. 313-345.

55. Seman, S.; Stockli, D.F.; McLean, N.M. U-Pb geochronology of grossular-andradite garnet. Chem. Geol. 2017, 460, 106-116. [CrossRef]

56. Wafforn, S.; Seman, S.; Kyle, J.; Stockli, D.; Leys, C.; Sonbait, D.; Cloos, M. Andradite garnet U/Pb geochronology of the Big Gossan Skarn, Ertsberg-Grasberg Mining District, Indonesia. Econ. Geol. 2018, in press.

57. Dziggel, A.; Wulff, K.; Kolb, J.; Meyer, F.M.; Lahaye, Y. Significance of oscillatory and bell-shaped growth zoning in hydrothermal garnet: Evidence from the Navachab gold deposit, Namibia. Chem. Geol. 2009, 262, 278-292. [CrossRef]

58. Xiao, X.; Zhou, T.; White, N.C.; Zhang, L.; Fan, Y.; Wang, F.; Chen, X. The formation and trace elements of garnet in the skarn zone from the Xinqiao Cu-S-Fe-Au deposit, Tongling ore district, Anhui Province, Eastern China. Lithos 2018, 302-303, 467-479. [CrossRef]

59. Bau, M. Rare-earth element mobility during hydrothermal and metamorphic fluid-rock interaction and the significance of the oxidation state of europium. Chem. Geol. 1991, 93, 219-230. [CrossRef]

60. Meinert, L.D. Skarns and skarn deposits. Geosci. Can. 1992, 19, 145-162.

61. Allen, D.E.; Seyfried, W.E. REE controls in ultramafic hosted MOR hydrothermal systems: An experimental study at elevated temperature and pressure. Geochim. Cosmochim. Acta 2005, 69, 675-683. [CrossRef]

(c) 2018 by the authors. Licensee MDPI, Basel, Switzerland. This article is an open access article distributed under the terms and conditions of the Creative Commons Attribution (CC BY) license (http:/ / creativecommons.org/licenses/by/4.0/). 